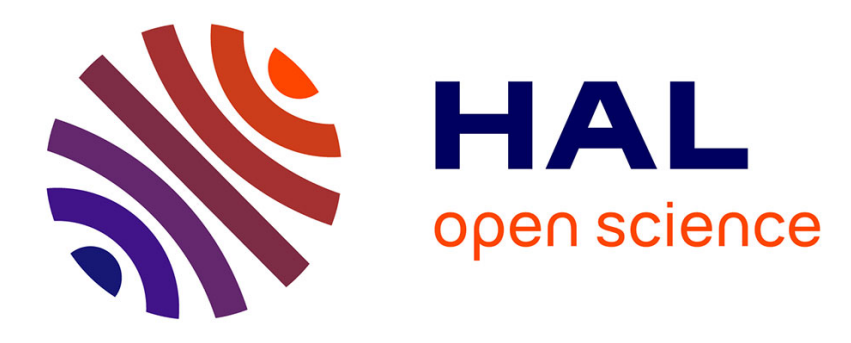

\title{
Rayleigh scattering in elastic composite materials
}

\author{
C. Boutin, J.L. Auriault
}

\section{To cite this version:}

C. Boutin, J.L. Auriault. Rayleigh scattering in elastic composite materials. International Journal of Engineering Science, 1993, 31 (12), pp.1669-1689. hal-00940461

\section{HAL Id: hal-00940461 \\ https://hal.science/hal-00940461}

Submitted on 1 Feb 2014

HAL is a multi-disciplinary open access archive for the deposit and dissemination of scientific research documents, whether they are published or not. The documents may come from teaching and research institutions in France or abroad, or from public or private research centers.
L'archive ouverte pluridisciplinaire $\mathbf{H A L}$, est destinée au dépôt et à la diffusion de documents scientifiques de niveau recherche, publiés ou non, émanant des établissements d'enseignement et de recherche français ou étrangers, des laboratoires publics ou privés. 


\title{
RAYLEIGH SCATTERING IN ELASTIC COMPOSITE MATERIALS
}

\author{
C. BOUTIN \\ Ecole Nationale des Travaux Publics de l'Etat, Laboratoire Géomatériaux DGCB, C.N.R.S. URA \\ 1652, Rue Maurice Audin, 69518 Vaulx-en-Velin Cedex, France \\ J. L. AURIAULT \\ Institut de Mécanique de Grenoble, 3S. Université Joseph Fourier, Institut National Polytechnique de \\ Grenoble, C.N.R.S. URA 1511, B.P. 53 X, 38041 Grenoble Cedex, France

\begin{abstract}
This paper is devoted to long wave propagation in heterogeneous media. More specifically, we deal with Rayleigh diffraction in elastic materials with a periodic microstructure whose heterogeneities are in finite concentration or show great contrasts in properties.

This study is based on the homogenization method but contrary to the usual procedure in which only the first significant terms are used, the developments are established up to the third order. We demonstrate that the terms of a superior order successively introduce effects of polarization, of celerity dispersion and of attenuation and we thus bring to the fore a characteristic distance of mode conversion. Finally we demonstrate that the effect of dispersion alone appears in macroscopically isotropic materials.
\end{abstract}

\section{INTRODUCTION}

The scattering of waves which propagate into heterogeneous materials is a well known phenomenon in the experimental field especially in the field of ultrasonics applied to the study of mechanical properties and/or the texture of materials with a crystalline structure (metals, rocks) or granular structure (porous medium, concrete) (for example [Laszlo et al., 1980]). In geophysics, the scattering has an important role to play too because it filters the waves that propagate into natural stratified soils or media that show cracks or cavities.

The essential parameter in the physical description of scattering (or diffraction) is the wave number $k$ multiplied by the characteristic dimension $l$ of heterogeneities. Classically we can distinguish three domains: the Rayleigh domain $(k l \ll 1)$, the intermediary or stochastic domain $(k l \approx 1)$ and the high frequencies domain $(k l \gg 1)$.

From a theoretical point of view, many surveys have been done on this subject and there are different methods that deal with this problem according to the domain taken into account (Born approximation, Bloch waves [Turbe, 1982],...). In [Gubernatis et al., 1977] and [Bond, 1989] a review of these approaches and of their domain of validity is given.

When dealing with long wave lengths, theoretical expressions allowing to quantify the effects of Rayleigh scattering are either available in the case of weak concentration of hererogeneities (for example [Piau, 1980]) or in the case of a quasi-homogeneous medium [Hirsekorn, 1988], [Stanke and Kino, 1984]. In the first case the results are obtained from the expansion of the fields radiated by the heterogeneities considered as infinitely distant from one another. In the second case Born approximation is used. Such an approximation is based on the expansion of elastic constants whose variations are very weak.

We put forward here a new approach to the Rayleigh scattering that allows us to deal from a theoretical point of view, with periodic microstructure materials whose heterogeneities are in finite concentration and show great contrasts in properties (for example a composite material or a medium with many cracks). We have therefore used the homogenization technique which was started and developed in [Bensoussan et al., 1978] and [Sanchez Palencia, 1980].

Such a very general method allows to "construct" the macroscopic behaviour of a heterogeneous medium. It is applicable when the characteristic macroscopic dimension $L$ is large when compared to the dimension $l$ of heterogeneities. Actually it is based on the 
asymptotical expansion of variables according to the powers of the small parameter $l / L$. Many problems especially the mechanics of composites [S, 1980], [Auriault and Bonnet, 1985], have been solved with this method. However, contrary to the usual procedure in which only the first significant term is taken into account, we establish developments up to the third order, which allows to describe most of the effects of the scattering [Boutin, 1991].

The role of these terms of superior order has been already investigated in statics in [Gambin and Kröner, 1989]. There, it is shown that the successive gradients of strain intervene in the macroscopic behaviour law.

In the second part, after having mentioned the principles of homogenization method we give the macroscopic balance equations that describe the dynamic behaviour of the heterogeneous medium. In order to obtain a more precise description, the analysis is carried through to the third order.

In the third part, we study the wave propagation while taking into account the balance equations at different orders. At zero order we find that the propagation is the one which occurs in an elastic homogeneous medium. But let's consider the terms of superior order, we then notice that order 1 introduces a correction of polarization, order 2 a dispersive effect on the celerity and third order is associated to an attenuation effect. These results are in agreement with the usual assessments of the scattering effects and moreover they give us a theoretical means to calculate all the effective coefficients from the knowledge of microstructure. Finally, this method allows us to demonstrate clearly that the polarization and attenuation effects appear only if the material shows an anisotropic macroscopic behaviour.

\section{ELASTODYNAMICS OF ELASTIC COMPOSITES}

Here we look into the dynamics of materials that show a periodic microstructure with an elastic behaviour (see Fig. 1). The method used is the homogenization of which we will briefly mention the basic principles. We use this method in paragraph 2.2 modifying the developments of $[G, 1989]$ which are valid in statics. This takes us to equations governing the dynamics of elastic composites, up to the third order.

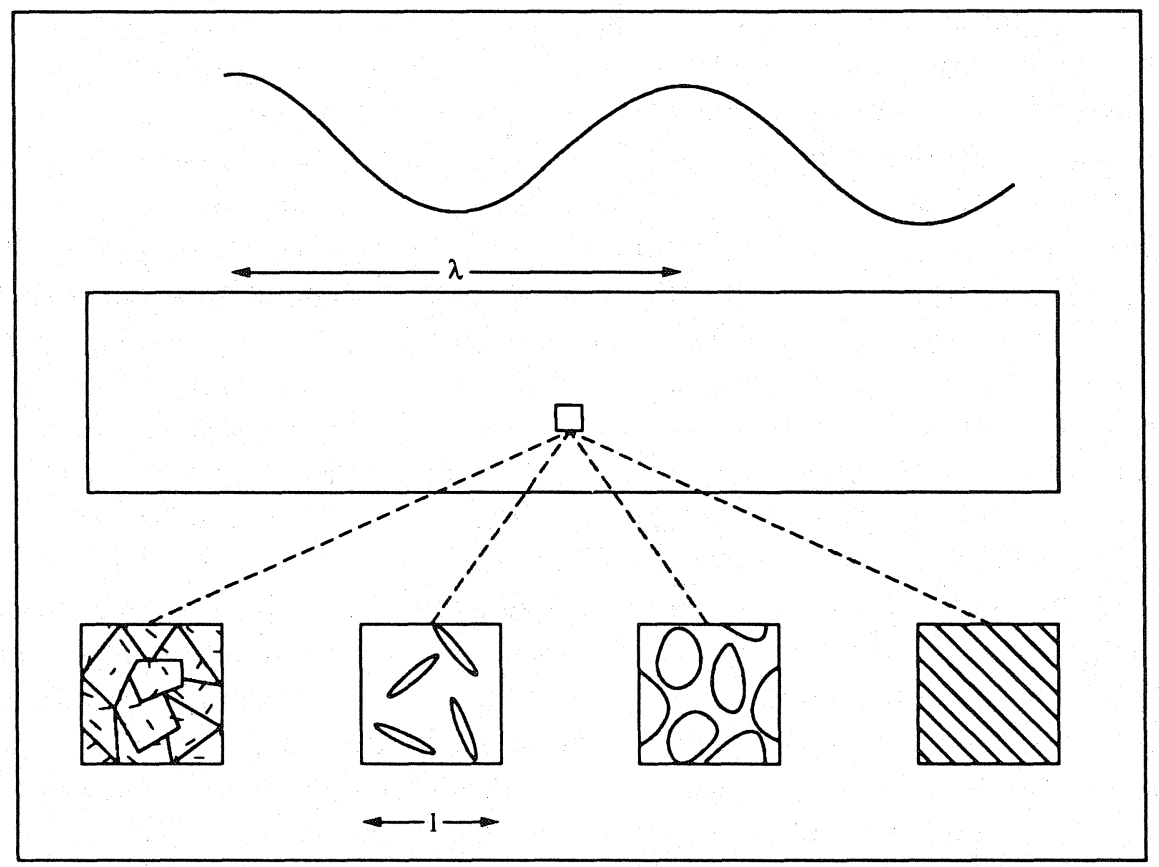

Fig. 1. Geometric configuration. Macroscopic wave length. Four examples of possible microscopic cells (polycrystalline, fissured, granular and composite materials). 


\subsection{Principle of the homogenization method}

The grounds of homogenization were established in [B, 1978] and [S, 1980]. This method allows to construct the macroscopic behaviour of a finitely heterogeneous periodic medium from the physical description of phenomena at the microscopic level. The fundamental assumption allowing to "replace" the initial material by an artificial medium macroscopically equivalent, is that the characteristic size of the macroscopic problem $L$ is very large compared to the dimension of the heterogeneities. In order to really be able to define two scales, the medium necessarily has to show an invariancy property by spatial translation. That is why we consider periodic media. $l$ being the characteristic length of the cell we must have $\varepsilon=l / L \ll 1$.

2.1.1 Double scale and asymptotical expansions. In order to mathematically transcribe the existence of these two well distinct scales, we will introduce two spatial variable systems, $x$ and $y$ with $y=\varepsilon^{-1} x$. Every quantity is therefore found in the form of a function of $x$ and $y$ in which $x$ describes the variations at the macroscopic level, whereas $y$ describes the local microscopic fluctuations. The use of such a double spatial variable system changes the usual derivatives $\partial_{X}$ to $\partial_{x}+\varepsilon^{-1} \partial_{y}$. This therefore introduces powers of $\varepsilon$ in the equations that describe physics on the local scale.

It thus seems natural to seek the physical quantities in the form of developments according to the powers of $\varepsilon$. For instance, for the displacements we will set down:

$$
U(x, y)=\sum_{0}^{\infty} \varepsilon^{i} U^{i}(x, y)
$$

Moreover, the periodic invariancy is conveyed by the periodicity according to the variables $y$ of each function at each order.

2.1.2 Homogenization process. The method consists in introducing the asymptotical expansions into the equations that describe the physics at the level of the cell, in identifying the powers of $\varepsilon$ and in successively solving the corresponding differential systems.

2.1.3 The small parameter $\varepsilon$. From a mathematical point of view, $\varepsilon$ is an infinitesimal quantity that allows to solve separately the problems associated to different orders.

From a physical point of view, things are different because the micro and macroscopic dimensions are finite and the parameter $\varepsilon$ is necessarily a finite quantity. Consequently, the macroscopic descriptions obtained at order $\varepsilon^{i}$ are not to be taken as correct representations except for when to the precision $\varepsilon^{i+1}$. Therefore the closer $\varepsilon$ is to 1 , the less the description is precise. It is thus important to know an evaluation of this parameter. For a given material, the size of the heterogeneities is fixed, but the macroscopic dimension depends on the solicitation. Particularly in the case of dynamics it can be shown that $L=\lambda / 2 \pi$ ( $\lambda$ : length of macroscopic wave) [Boutin and Auriault, 1990], which gives $\varepsilon=\omega l / V(V$ : celerity of the wave).

Later, we will use these two aspects of this parameter: the mathematical definition to establish macroscopic equations, the physical approach to specify an approximation of the different terms and the accuracy of the description that has been obtained.

\subsection{Homogenization of elastic microstructures}

Let's consider a periodic medium made of cells $\Omega$ of the characteristic dimension $l$ (see Fig. 1). These cells are made of elastic material whose elastic tensor $c(y)$ and density $\rho(y)$ vary and can be discontinuous in the period $\Omega$. We take the case of an important contrast in properties. That is to say that contrary to the usual assumption of Born approximation the variations of $c$ and $\rho$ are not small fluctuations but of the same order of magnitude as their average value. Moreover we assume that the dimensions of heterogeneities are similar to the dimension of the cell (heavy concentration) and that consequently their interactions are not to be left unnoticed.

We study in the Rayleigh domain, and in the hypothesis of small deformations, the propagation of harmonic waves at pulsation $\omega=2 \pi f$. In such conditions, length $\lambda$ of 
macroscopic wave is very large when compared to $l$, so that $\varepsilon=2 \pi l / \lambda \ll 1$ and that the homogenization method is applicable.

2.2.1 Basic equations. On the scale of the cell, the motion is described by the elastodynamic equations:

$$
L_{y}(u(y))=\nabla_{y} . c(y) . . e_{y}(u(y))+\omega^{2} \rho(y) u(y)=0
$$

In this expression, $u(y)$ represents the field of displacement and the dot means contraction (.. means double contraction and ... triple contraction etc.). $\nabla_{y}$ and $e_{y}$ are respectively the Nabla operator and deformation operator that act on the variables $y$. Later, $\nabla_{x}$ and $e_{x}$ are the same operators that act on the variables $x$.

As has been described earlier, we introduce the double spatial coordinates $x$ and $y$ (in which $\left.y=\varepsilon^{-1} x\right)$ with $L=\lambda / 2 \pi$ as for reference length $(l=\varepsilon l)$. Then taking the dimensional analysis into account, the initial operator changes to $[\mathrm{G}, 1989]$ :

with:

$$
L_{x, y}(-)=\varepsilon^{-2} L^{-2}(-)+\varepsilon^{-1} L^{-1}(-)+L^{0}(-)
$$

$$
\begin{aligned}
L^{-2}(-) & =\nabla_{y} . c(y) . . e_{y}(-) \\
L^{-1}(-) & =\nabla_{y} . c(y) . . e_{x}(-)+\nabla_{x} . c(y) . . e_{y}(-) \\
L^{0}(-) & =\nabla_{x} . c(y) . . e_{x}(-)+\omega^{2} \rho(y)(-)
\end{aligned}
$$

As far as the boundary conditions are concerned, the field of displacement must be continuous and so must the stress vector $\sigma . n$ on the possible discontinuities surfaces of the elastic tensor $c(y)$ (in this case the derivative have to be taken in the frame of distribution theory). As a result of the double scale, $\sigma$ is expressed in the following form:

$$
\sigma=c(y) . .\left(\varepsilon^{-1} e_{y}(U)+e_{x}(U)\right)
$$

2.2.2 Homogenization process. We only give here the main lines of the reasoning. But for the modification added by the inertial terms, the calculations are similar to those proposed in the static case in [G, 1989]. The details of the homogenization process are given in Appendix 1.

When introducing the asymptotical expansion (1) of displacement $U$ in operator $L_{x, y}$, a series according to the power of $\varepsilon$ is obtained. As this series must be identically equal to zero, each of its terms are equal to zero. All the same we assume that the continuity of the displacements and stress vector is verified by each term of their expansion. This leads us to the following series of problems set down in the cell and dealing with the $\Omega$-periodic functions $U^{i}$ :

$$
\begin{aligned}
& L^{-2}\left(U^{0}\right)=0 \\
& L^{-2}\left(U^{1}\right)=-\left[L^{-1}\left(U^{0}\right)\right]
\end{aligned}
$$

and for $i \geq 0$ :

$$
L^{-2}\left(U^{i+2}\right)=-\left[L^{-1}\left(U^{i+1}\right)+L^{0}\left(U^{i}\right)\right]
$$

These problems being linear, they are dealt with from their variational formulation. Their solving is done successively: once the terms $U^{i}$ and $U^{i+1}$ have been determined, they become forcing terms in the solving of the problem relating to $U^{i+2}$, and so on...

On the other hand, the equations (2.i) can also be expressed in their equivalent form in which the stresses at two successive orders intervene:

with:

$$
\nabla_{y} . \sigma^{i+1}+\nabla_{x} . \sigma^{i}+\omega^{2} \rho U^{i}=0
$$

$$
\sigma^{i}=c(y) . .\left(e_{y}\left(U^{i+1}\right)+e_{x}\left(U^{i}\right)\right)
$$

Because of the continuity and the periodicity of the stresses the integration of this equation on the period (according to the variable $y$ ), allows to eliminate the first term and gives the following relation, which links the fields of displacement and of stress at the same order 
(equation of compatibility):

$$
\nabla_{x} \cdot \int_{\Omega} \sigma^{i} \mathrm{~d} \Omega+\omega^{2} \int_{\Omega} \rho U^{i} \mathrm{~d} \Omega=0
$$

When dividing by the volume of the period and-in order to simplify the writing-putting a bar over the averaged terms, we obtain:

$$
\nabla_{x} \cdot \overline{\sigma^{i}}+\omega^{2} \overline{\rho U^{i}}=0
$$

These relations are very important because they express the balance of all the stresses that act on the cell at order $\varepsilon^{i}$. Generally we are satisfied with the equation at order zero. If a more precise description is needed, as it is the case for the scattering, the superior orders have to be taken into account.

2.2.3 Macroscopic description of homogenized medium. The homogenization process leads to a field of displacement of the following sort:

$$
U(x, y)=U^{0}(x)+\sum_{1}^{\infty} \varepsilon^{i} U^{i}(x, y)
$$

in which each term of the sum is split up into a constant in the cell and into a sum of terms that vary in the period. These latter terms have a cell average equal to zero.

$$
U^{i}(x, y)=\overline{U^{i}}(x)+\sum_{k=0}^{i-1} U^{i k}(x, y)
$$

Each of these local fields is associated to successive gradients of macroscopic strain tensor of constant displacements of an inferior order:

So:

$$
U^{i k}(x, y)=X^{k+1}(y) \cdot\left(. \nabla_{x}\right)^{k} e_{x}\left(\overline{U^{i-k-1}}(x)\right)
$$

$$
U^{i}(x, y)=\overline{U^{i}}(x)+\sum_{k=0}^{i-1} X^{k+1}(y) \cdot\left(\nabla_{x}\right)^{k} e_{x}\left(\overline{U^{i-k-1}}(x)\right)
$$

The first four equations of macroscopic balance given below allow to describe the average displacement up to order 3 . The macroscopic field really observable is obtained by the addition of the different orders:

$$
\bar{U}(x)=\overline{U^{0}}(x)+\varepsilon \overline{U^{1}}(x)+\varepsilon^{2} \overline{U^{2}}(x)+\varepsilon^{3} \overline{U^{3}}(x)
$$

Order 0:

$$
\nabla_{x} \cdot \overline{C^{0}} . . e_{x}\left(\overline{U^{0}}\right)+\omega^{2} \bar{\rho} \overline{U^{0}}=0
$$

Order 1:

$$
\nabla_{x} \cdot \overline{C^{0}} . . e_{x}\left(\overline{U^{1}}\right)+\omega^{2} \bar{\rho} \overline{U^{1}}=-\left[\nabla_{x} \cdot \overline{C^{1}} \ldots \nabla_{x} e_{x}\left(\overline{U^{0}}\right)+\omega^{2} \overline{\rho X^{1}} . . e_{x}\left(\overline{U^{0}}\right)\right]
$$

Order 2:

$$
\begin{aligned}
\nabla_{x} . \overline{C^{0}} . . e_{x}\left(\overline{U^{2}}\right)+\omega^{2} \bar{\rho} \overline{U^{2}}= & -\left[\nabla_{x} \cdot \overline{C^{1}} \ldots \nabla_{x} e_{x}\left(\overline{U^{1}}\right)+\omega^{2} \overline{\rho X^{1}} . . e_{x}\left(\overline{U^{1}}\right)\right] \\
& -\left[\nabla_{x} \cdot \overline{C^{2}} \ldots . \nabla_{x} \nabla_{x} e_{x}\left(\overline{U^{0}}\right)+\omega^{2} \overline{\rho X^{2}} \ldots \nabla_{x} e_{x}\left(\overline{U^{0}}\right)\right]
\end{aligned}
$$

Order 3:

$$
\begin{aligned}
\nabla_{x} \cdot \overline{C^{0}} . . e_{x}\left(\overline{U^{3}}\right)+\omega^{2} \bar{\rho} \overline{U^{3}}= & -\left[\nabla_{x} \cdot \overline{C^{1}} \ldots \nabla_{x} e_{x}\left(\overline{U^{2}}\right)+\omega^{2} \overline{\rho X^{1}} . . e_{x}\left(\overline{U^{2}}\right)\right] \\
& -\left[\nabla_{x} \cdot \overline{C^{2}} \ldots . \nabla_{x} \nabla_{x} e_{x}\left(\overline{U^{1}}\right)+\omega^{2} \overline{\rho X^{2}} \ldots \nabla_{x} e_{x}\left(\overline{U^{1}}\right)\right] \\
& -\left[\nabla_{x} \cdot \overline{C^{3}} \ldots . . \nabla_{x} \nabla_{x} \nabla_{x} e_{x}\left(\overline{U^{0}}\right)+\omega^{2} \overline{\rho X^{3}} \ldots \nabla_{x} \nabla_{x} e_{x}\left(\overline{U^{0}}\right)\right]
\end{aligned}
$$

Later, we will write these balance equations in the following condensed form:

$$
\begin{gathered}
S^{0}\left(\overline{U^{0}}\right)=0 \\
S^{0}\left(\overline{U^{1}}\right)=-\left[S^{1}\left(\overline{U^{0}}\right)\right]
\end{gathered}
$$




$$
\begin{gathered}
S^{0}\left(\overline{U^{2}}\right)=-\left[S^{1}\left(\overline{U^{1}}\right)+S^{2}\left(\overline{U^{0}}\right)\right] \\
S^{0}\left(\overline{U^{3}}\right)=-\left[S^{1}\left(\overline{U^{2}}\right)+S^{2}\left(\overline{U^{1}}\right)+S^{3}\left(\overline{U^{0}}\right)\right]
\end{gathered}
$$

2.2.4 Remarks on the effective tensors $\overline{C^{i}}$ and $\overline{\rho X^{i}}$. The different tensors $\overline{C^{i}}, \overline{\rho X^{i}}$ appearing in these equations are all entirely determined from the knowledge of $c(y)$ and $\rho(y)$ on the period. They are constructed from particular solutions of statics problems that are set down in the cell. That is why they are independant of the solicitation frequency. The theoretical equations that allow us to calculate them are given in Appendix 1.

$\overline{C^{0}}$ is the fourth rank tensor which characterizes the macroscopic behaviour of the equivalent elastic material. It depends entirely on $c(y)$ and is of the same order of magnitude as $c(y)$. It has been shown in $[S, 1980]$ that $\overline{C^{0}}$ verifies all the classic properties of elastic tensors.

The tensors $\bar{C}$ are different from the usual ones since they are of rank $i+4$ and are homogeneous to $\operatorname{Pa}(m)^{i}$. Moreover, they depend both on $c(y)$ and $\rho(y)$ because they result from the stresses and the inertial forces associated to the local displacements. As far as the order of magnitude is concerned, and since these tensors appear in the equations only in the form of the product $\varepsilon^{i} \overline{C^{i}}$, this latter term is the one that has to be assessed. As $L$ and $c$ are respectively the length and the modulus of reference, $\varepsilon^{i} \overline{C^{i}}$ is of the order of $\varepsilon^{i} c(L)^{i}=c(l)^{i}$. Finally, we notice that when the density is constant on the period, these tensors coincide with those that have been obtained in [G, 1989].

The tensors $\overline{\rho X^{i}}$ of rank $i+2$ are homogeneous to $\mathrm{kg}(\mathrm{m})^{i-3}$. They are associated to the inertial terms of the local fields and also depend on $c(y)$ and $\rho(y)$. As precedingly the terms $\varepsilon^{i} \overline{\rho X^{i}}$ are those to be assessed. We simply have: $O\left(\varepsilon^{i} \overline{\rho X^{i}}\right)=\rho(l)^{i}$. Let's notice that these tensors are equal to zero when the density is constant on the period.

In the tensor theory, it is known that the tensors of odd rank are necessarily anisotropic. So the terms $S^{i}$ which use $\overline{C^{i}}$ and $\overline{\rho X^{i}}$, (i being odd) necessarily reveal a macroscopic anisotropy of the homogenized medium. In the case of a macroscopically isotropic medium, these terms are equal to zero. Let us specify that this assumption seems hardly feasible with periodic materials whose size of heterogeneities is of the same order of magnitude as the period's.

2.2.5 Comments on the macroscopic balance equations. In the momentum balance of zero order, $S^{0}$ is the classic operator of elastodynamics for a homogeneous medium of elasticity tensor $\overline{C^{0}}$ and of density $\bar{\rho}$. According to the structure of the period, this homogenized material can either be isotropic or anisotropic.

Contrary to what would happen for a really homogeneous material (that is to say that doesn't show any heterogeneity on any scale), in the balance equations of superior order, there appear new operators $S^{i}$, of order $2+i$, which use, as in the statics case, the successive deformation gradients. These equations at order $i>0$, correspond to the momentum equation in a homogeneous medium of properties $\overline{C^{0}}$ and $\bar{\rho}$, submitted to a field of force density directly resulting from fields of inferior orders $j<i$. Thus, when in the presence of heterogeneities, a field of order zero creates a force density of order 1 which generates a field of order 1 . These two fields, through the same process, produce a volumic force which radiates a field at order 2 , and so on...

We are back to the phenomenon of the Rayleigh scattering in which the passing of a long wave through heterogeneities generates sources that perturb the initial wave. The interest of the homogenization method is to "replace" these sources and their "precise" radiation by "homogenized" sources and fields that are equivalent, at the considered order.

\section{STUDY OF THE SCATTERING OF A PLANE WAVE}

In this part we look into the incidence of terms of superior order that have been introduced precedingly, on a macroscopic plane wave propagating in any direction $p$. From an energy point of view, we are interested in the energy flux carried in direction $p$. 
In a first time, we mention the propagation in a homogeneous anisotropic elastic medium (zero order). We then show that the perturbation caused by the heterogeneities are of a different nature according to the order of the correcting terms considered (polarization, dispersion and attenuation). Finally, the analysis of the conditions of validity of the description that has been obtained, allows to give a rough idea as to the distance of non-perturbation of the wave, beyond which there will be mode conversions.

\subsection{Order 0: propagation in an elastic homogeneous medium}

In what follows, as we now work at the macroscopic level, we will not put bars over the averaged quantities (we write $U^{i}, C^{i}, \rho$ instead of $\bar{U}^{i}, \overline{C^{i}}, \bar{\rho}$ ) and in order to simplify the writing we write $R^{i}$ instead of $\overline{\rho X^{i}}$. We also suppress the suffix $x$ for the macroscopic operators and we simply write $x$ for the variable $x_{1}$.

Let's consider an anisotropic medium homogenized at order zero, in which an harmonic plane wave propagates in any direction $p$. So as to simplify the calculations-without putting limits to the scope of the reasoning - we switch to a one dimensional problem. In order to achieve it we assume that the equations (and the tensors to which we give the index $p$ ) are expressed in an orthonormal frame $E_{p}$ whose direction of the first axes $o x_{1}$ coincides with $p$.

In the frame $E_{p}$, we look for the plane waves of the following form:

$$
U^{0}=A \exp (-i k x) \exp (i \omega t)
$$

The first momentum balance (3.0), in an indicial form, leads to the classic dispersion equation:

$$
-k^{2}\left(C_{p i 1}^{01 j} A_{j}\right)+\omega^{2} \rho A_{i}=0
$$

(see Appendix 1 for the index conventions). In a matricial form:

$$
k^{2} M_{p} A=\omega^{2} \rho A \quad M_{p i j}=C_{p i 1}^{01 j}
$$

From the properties of elastic tensor $C^{0}$ the matrix $M_{p}$ is symmetrical, defined and positive. We can deduce from it that in the direction considered, there are three modes of propagation with three distinct directions of polarization (see Fig. 2). The directions of polarization $D^{a}, D^{b}, D^{c}$, are the eigenvectors of $M_{p}$. Three real positive eigenvalues $N_{i}$ of $M_{p}$ are associated to the vectors $D^{i}$. They are linked to the wave number $k_{i}$ by the relation:

$$
N_{i}\left(k_{i}\right)^{2}=\omega^{2} \rho
$$

which leads to the celerities $V_{i}$ :

$$
V_{i}=\frac{\omega}{k_{i}}=\sqrt{N_{i} / \rho}
$$

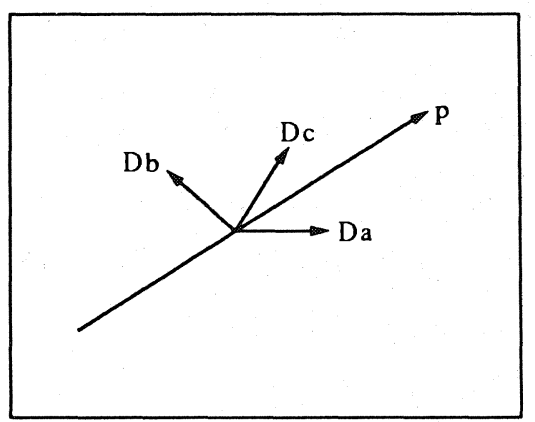

Fig. 2. The three polarization directions of waves propagating in direction $p$. 
It is obvious that since $M_{p}$ depends on the chosen direction $p$, the characteristics of the modes also depend on it. There we are back to the usual results concerning the propagation of plane waves in an anisotropic homogeneous elastic medium.

Later, we assume that at order zero, a "mode $a$ " wave of unit amplitude propagates. That is to say that we have:

$$
U^{0}=D^{a} \exp \left(-i k_{a} x\right) \exp (i \omega t)
$$

\subsection{Taking terms of superior order into account}

The description of mechanical waves given above is only correct to the precision $\varepsilon$. In the case of very long wave lengths, such a precision is sufficient. On the contrary when we consider wave lengths only 10 to 100 times superior to the size of the heterogeneities, it is interesting to give more precision as to the analysis of the propagation through considering the terms of higher order.

For a given order we will calculate the volume sources $S^{i}\left(U^{j}\right)(i>0)$, that result from wave fields at inferior orders $(j<i)$. This allows us to turn to the field radiated by its sources. We obtain the macroscopically observable wave field when adding the fields at different orders.

We notice that as these problems are linear, all the fields oscillate at the same frequency: we therefore suppress the term $\exp (i \omega t)$.

3.2.1 Order 1: correction of polarization. Equation (4.1) of the momentum balance at order 1 show that $U^{1}$ is the sum of any field, solution of the homogeneous problem (but without any interest in our case) and of the field radiated by the source distribution $-S^{1}\left(U^{0}\right)$, with:

$$
-S^{1}\left(U^{0}\right)=-\left[\nabla . C^{1} \ldots \nabla e\left(U^{0}\right)+\omega^{2} R^{1} . . e\left(U^{0}\right)\right]
$$

Let us give the explicit expression of $-S^{1}\left(U^{0}\right)$. Using the index notation we have:

$$
-S_{i}^{1}\left(U^{0}\right)=-\left[C_{p i j}^{1 k l m} U_{m, l k j}^{0}+\omega^{2} R_{p i}^{1 q r} U_{r, q}^{0}\right]
$$

and, when introducing the expression (5) of $U^{0}$ :

$$
-S_{i}^{1}\left(U^{0}\right)=i k_{a}\left[\left(-i k_{a}\right)^{2} C_{p i 1}^{111 m} D_{m}^{a}+\omega^{2} R_{p i}^{11 r} D_{r}^{a}\right] \exp \left(-i k_{a} x\right)
$$

which gives, in a matricial form:

$$
-S^{1}\left(U^{0}\right)=i k_{a} Q_{p}^{1} D^{a} \exp \left(-i k_{a} x\right) \quad Q_{p i j}^{1}=\left(-i k_{a}\right)^{2} C_{p i 1}^{111 j}+\omega^{2} R_{p i}^{11 j}
$$

$D^{a}$ being a propagation mode, we can also write:

$$
Q_{p i j}^{1} D_{j}^{a}=\left(-i k_{a}\right)^{2}\left(C_{p i 1}^{1 j 11}-R_{p i}^{11 k} C_{p k 1}^{0 j 1} \rho^{-1}\right) D_{j}^{a}
$$

We notice that the volume sources are identical in every point of the planes parallel to the wave front. On the one hand, these sources oscillate spatially according to the wave length of the mode $a$ and in quadrature of phase with it. On the other hand, we demonstrate that these volumic forces are orthogonal to the polarization vector $D^{a}$. The details of the calculations are given in Appendix 2. The whole of these considerations leads us to look for $U^{1}$ in the following form:

$$
U^{1}=\left(b_{1} D^{b}+c_{1} D^{c}\right) i k_{a} \exp \left(-i k_{a} x\right)
$$

When applying operator $S^{0}$ to this expression, we obtain in a matricial form:

$$
S^{0}\left(U^{1}\right)=\left[\left(-i k_{a}\right)^{2} M_{p}+\omega^{2} \rho I\right]\left(b_{1} D^{b}+c_{1} D^{c}\right) i k_{a} \exp \left(-i k_{a} x\right)
$$

Then, as $D^{b}$ and $D^{c}$ are eigenvectors of $M_{p}$ :

$$
S^{0}\left(U^{1}\right)=\omega^{2} \rho\left(\left(1-\left[\frac{k_{a}}{k_{b}}\right]^{2}\right) b_{1} D^{b}+\left(1-\left[\frac{k_{a}}{k_{c}}\right]^{2}\right) c_{1} D^{c}\right) i k_{a} \exp \left(-i k_{a} x\right)
$$


Once identified with the source term, the set $\left\{D^{i}\right\}$ being orthonormal, we deduce the expressions of real coefficients $b_{1}$ and $c_{1}$ :

$$
b_{1}=D^{b} \cdot Q_{p}^{1} \cdot D^{a}\left[\omega^{2} \rho\left(1-\left[k_{a} / k_{b}\right]^{2}\right)\right]^{-1} \quad c_{1}=D^{c} \cdot Q_{p}^{1} \cdot D^{a}\left[\omega^{2} \rho\left(1-\left[k_{a} / k_{c}\right]^{2}\right)\right]^{-1}
$$

Finally, at order 1 the global macroscopic field is:

$$
U(x)=U^{0}(x)+\varepsilon U^{1}(x)=\left[D^{a}+\varepsilon\left(b_{1} D^{b}+c_{1} D^{c}\right) i k_{a}\right] \exp \left(-i k_{a} x\right)
$$

As a conclusion we notice that the global wave is not any longer rigorously polarized according to $D^{a}$. On the contrary the taking into account of the diffracted field at the first order does not modify the wave length nor the direction of the propagation. Thus, this deviation of polarization does not introduce any mode conversion (see paragraph 3.3.3). As this correction of polarization is in quadrature of phase the macroscopic trajectory of the particles is ellipsoidal (see Fig. 3). It is possible to check that the amplitude of these supplementary terms is of order 1 by evaluating $\varepsilon b_{1} k_{a}$ and $\varepsilon c_{1} k_{a}$. From the expressions of $b_{1}$ and $c_{1}$ :

$$
O\left(b_{1}\right)=O\left(c_{1}\right)=O\left(Q_{p}^{1} / \omega^{2} \rho l\right)
$$

But with the estimations of $C^{1}$ and $R^{1}$ :

$$
O\left(\varepsilon Q_{p}^{1}\right)=O\left(c l L^{2}\right)=O\left(\omega^{2} \rho l\right)
$$

And, as expected:

$$
O\left(\varepsilon b_{1} k_{a}\right)=O\left(\varepsilon c_{1} k_{a}\right) O\left(l k_{a}\right)=O(\varepsilon)=O\left(\omega l / V_{a}\right)
$$

The effect of polarization is therefore a phenomenon which depends linearily on the frequency.

3.2.2 Order 2: dispersion of celerity. In this paragraph, we look into the corrections brought by the terms of second order. We observe in equation (4.2) that the sources come from second order effects resulting from field $U^{0}$, i.e. $-S^{2}\left(U^{0}\right)$, and fields $U^{1}$, i.e. $-S^{1}\left(U^{1}\right)$. Let's calculate these volume forces.

As for the sources resulting from $U^{0}$, we have:

$$
-S^{2}\left(U^{0}\right)=-\left[\nabla . C^{2} \ldots \nabla \nabla e\left(U^{0}\right)+\omega^{2} R^{2} \ldots \nabla e\left(U^{0}\right)\right]
$$

That is to say, in the frame $E_{p}$ :

$$
-S_{i}^{2}\left(U^{0}\right)=-\left[C_{p i j}^{2 k l m n} U_{n, m l k j}^{0}+\omega^{2} R_{p i}^{2 q r s} U_{s, r q}^{0}\right]
$$

And, when introducing the expression (5) of $U^{0}$ :

$$
-S_{i}^{2}\left(U^{0}\right)=\left(k_{a}\right)^{2}\left[\left(-i k_{a}\right)^{2} C_{p i 1}^{2111 n} D_{n}^{a}+\omega^{2} R_{p i}^{211 s} D_{s}^{a}\right] \exp \left(-i k_{a} x\right)
$$

Which gives in a matricial form:

$$
-S^{2}\left(U^{0}\right)=\left(k_{a}\right)^{2} Q_{p}^{2} D^{a} \exp \left(-i k_{a} x\right) \quad Q_{p i j}^{2}=\left(-i k_{a}\right)^{2} C_{p i 1}^{2111 j}+\omega^{2} R_{p i}^{211 j}
$$

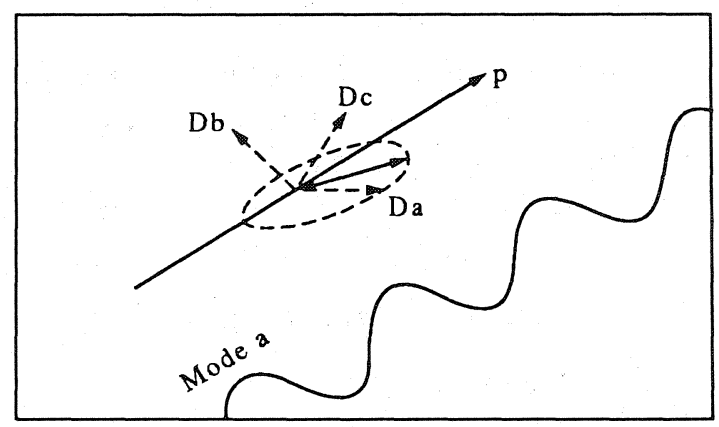

Fig. 3. Correction of polarization at the first order. 
As far as the source resulting from $U^{1}$ is concerned, we have:

$$
-S^{1}\left(U^{1}\right)=-\left[\nabla . C^{1} \ldots \nabla e\left(U^{1}\right)+\omega^{2} R^{1} . . e\left(U^{1}\right)\right]
$$

Which gives with the expression (6) of $U^{1}$ :

$$
-S^{1}\left(U^{1}\right)=\left(i k_{a}\right)^{2} Q_{p}\left(b_{1} D^{b}+c_{1} D^{c}\right) \exp \left(-i k_{a} x\right)
$$

Finally, we obtain:

$$
S^{0}\left(U^{2}\right)=-S^{2}\left(U^{0}\right)-S^{1}\left(U^{1}\right)=\left[Q_{p}^{2} D^{a}-Q_{p}\left(b_{1} D^{b}+c_{1} D^{c}\right)\right]\left(k_{a}\right)^{2} \exp \left(-i k_{a} x\right)
$$

As we have seen before, the field $U^{2}$ is constituted of:

-a field which is solution to the homogeneous problem (without any interest as far as the diffraction is concerned);

- and a particular field which results from $S^{2}\left(U^{0}\right)$ and $S^{1}\left(U^{1}\right)$. As for the first order, such a distribution of source respects the geometry of the "mode a" plane wave. On the contrary these elementary forces are not necessarily orthogonal to the direction of polarization $D^{a}$ of mode $a$. The component following $D^{a}$ will therefore come and excite the elastodynamic operator $S^{0}$ according to one of these eigen modes. This is expressed by a perturbation according to this mode, which is amplified as the wave progresses. We will then try to find $U^{2}$ in the following form:

$$
U^{2}=\left[\left(-i k_{a} x\right) a_{2} D^{a}+b_{2} D^{b}+c_{2} D^{c}\right]\left(k_{a}\right)^{2} \exp \left(-i k_{a} x\right)
$$

The application of operator $S^{0}$ to this expression gives in the matricial form:

$$
\left(\left[\left(-i k_{a}\right)^{2} M_{p}+\omega^{2} \rho I\right]\left[\left(i k_{a} x\right) a_{2} D^{a}+b_{2} D^{b}+c_{2} D^{c}\right]-2 a_{2}\left(k_{a}\right)^{2} M_{p} D^{a}\right)\left(k_{a}\right)^{2} \exp \left(-i k_{a} x\right)
$$

Then, $D^{a}, D^{b}$ and $D^{c}$ being the $M_{p}$ eigenvectors:

$$
S^{0}\left(U^{2}\right)=\omega^{2} \rho\left\{-2 a_{2} D^{a}+\left[\left(1-\left[\frac{k_{a}}{k_{b}}\right]^{2}\right) b_{2} D^{b}+\left(1-\left[\frac{k_{a}}{k_{c}}\right]^{2}\right) c_{2} D^{c}\right]\left(k_{a}\right)^{2}\right\} \exp \left(-i k_{a} x\right)
$$

By identification with the source term that has been calculated above, we can deduce the value of the real coefficients $a_{2}, b_{2}, c_{2}$. We can check, without having to calculate them, that the terms $\varepsilon^{2} a_{2} k a^{2}$ (or $\varepsilon^{2} b_{2} k_{a}$ or $\varepsilon^{2} c_{2} k_{a}$ ) are of order 2: If we take into account the different range of magnitude of the tensors $C^{i}$ and $X^{i}$ and the expression of the matrices $Q_{p}^{1}$ and $Q_{2}^{p}$ we have:

$$
O\left(\varepsilon^{2} Q_{p}^{2}\right)=O\left(c l^{2} L^{2}\right)=O\left(\omega^{2} \rho l^{2}\right)=O\left(\varepsilon^{2} b_{1} Q_{p}\right)
$$

And since:

we obtain:

$$
O\left(\omega^{2} \rho a_{2}\right)=O\left(Q_{p}^{2}\right)
$$

$$
O\left(\varepsilon^{2} a_{2} k_{a}^{2}\right)=O\left(\varepsilon^{2} b_{2} k_{a}\right)=O\left(\varepsilon^{2} c_{2} k_{a}\right)=O\left(k_{a}^{2} l^{2}\right)=O\left(\varepsilon^{2}\right)
$$

Thus, up to order 2 , the field of macroscopic wave is given by:

$$
\begin{aligned}
U(x)=U^{\prime}(x)+\varepsilon & \varepsilon U^{1}(x)+\varepsilon^{2} U^{2}(x) \\
& =\left\{\left(1-i \varepsilon^{2} A_{2} k_{a}^{2}\left(k_{a} x\right)\right) D^{a}+\left[\left(b_{1}-\varepsilon i k_{a} b_{2}\right) D^{b}+\left(c_{1}-\varepsilon i k_{a} c_{2}\right) D^{c}\right] \varepsilon i k_{a}\right\} \exp \left(-i k_{a} x\right)
\end{aligned}
$$

We can observe a new correction of polarization of order 2 , in quadrature of phase with the first one. Moreover, according to the direction of polarization, a term in quadrature of phase that linearily increases with $x$ perturbs the propagation (see Fig. 4). In order to look closely into the influence of this term we come back to the real expression. The wave amplitude according to $D^{a}$ is equal to:

$$
\alpha=\cos \left(k_{a} x\right)-\varepsilon^{2} a_{2} k_{a}^{2}\left(k_{a} x\right) \sin \left(k_{a} x\right)
$$

When introducing the phase shift $\varphi$ such as: $\operatorname{tg}(\varphi)=\varepsilon^{2} a_{2} k_{a}^{2}\left(k_{a} x\right)$

$$
\alpha=\cos \left(k_{a} x+\varphi\right)(\cos (\varphi))^{-1}
$$




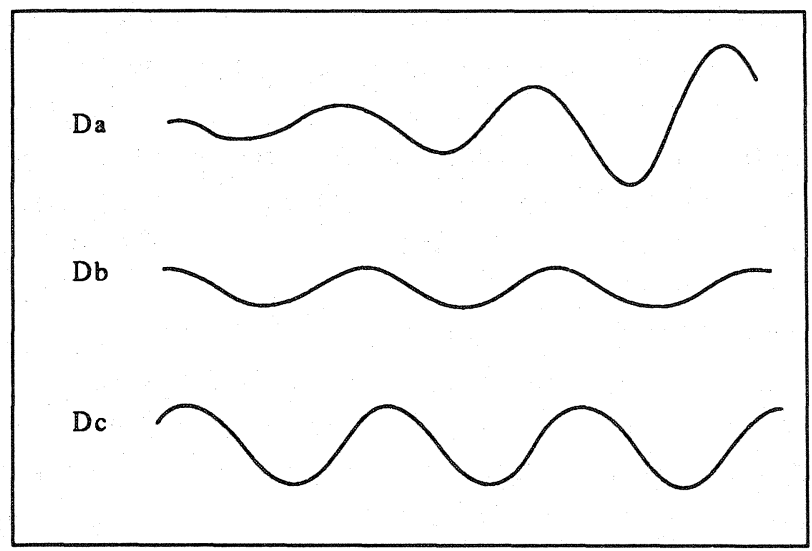

Fig. 4. Wave field at the second order: the vibration polarized in direction Da is amplified.

Let's look for a correct approximation of $\alpha$ up to order $\varepsilon^{2}$. All we have to do is to expand $\operatorname{tg}(\varphi)$. However, as $\operatorname{tg}(\varphi)$ is a function that linearily increases with $x$, we must keep within distances $x$ such as:

$$
O\left(k_{a} x\right) \leq \varepsilon^{-1 / 2}
$$

so: $0<x<\delta_{p}$, with: $\delta_{p}=\lambda / 2 \pi \sqrt{ } \lambda / 2 \pi l=\left(V_{a} / \omega\right)^{3 / 2} / \sqrt{ } l$.

Then, as $a_{2} k_{a}^{2}$ is $O(1)$ :

$$
\varphi=O\left(\varepsilon^{3 / 2}\right) \operatorname{tg}(\varphi) \cong \varphi\left(1+O\left(\varepsilon^{3}\right)\right) \text { and } \cos (\varphi) \cong 1+O\left(\varepsilon^{3}\right)
$$

We can therefore deduce that to $\varepsilon^{3}$, for $0<x<\delta_{p}$ :

$$
\alpha=\cos \left(k_{a}\left(1+\varepsilon^{2} a_{2} k_{a}^{2}\right) x\right)
$$

We conclude that the global component of the "mode $a$ " wave polarized according to $D^{a}$, propagates at a celerity which is slightly different from the one that has been calculated at zero order. This results from the "resonant" effect generated by the diffracted sources, and from constructive interferences between the diffracted waves and the wave at zero order (see Fig. 5).

Macroscopically, we can observe the celerity:

$$
V_{a}^{\prime}=\omega\left(k_{a}\left(1+\varepsilon^{2} a_{2} k_{a}^{2}\right)\right)^{-1}=V_{a}\left(1+\varepsilon^{2} a_{2} k_{a}^{2}\right)^{-1}
$$

The relative correction of celerity is of order $\varepsilon^{2}$ that is to say $O\left(\left(\omega l / V_{a}\right)^{2}\right.$. This effect varies therefore with the square of frequency. Let's notice that in spite of this dispersion, celerities $V_{a}$ and $V_{a}^{\prime}$ necessarily keep within the same range of magnitude (since $\varepsilon \ll 1$ ).

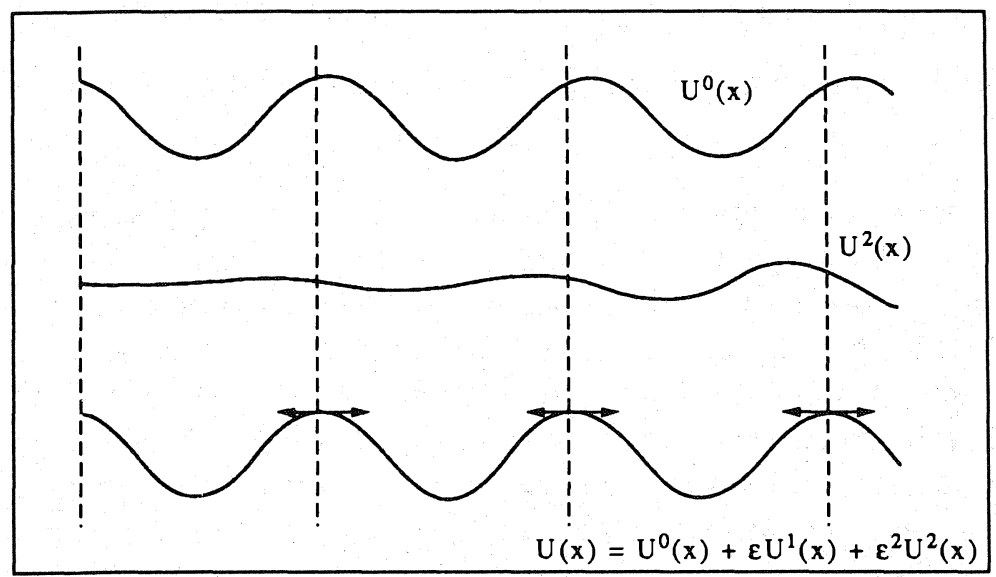

Fig. 5. The effect of dispersion results of the summation of the wave $U^{0}$ and the wave $U^{2}$ (in phase quadrature and amplified). 
The increasing or decreasing of the celerity with the frequency depends on the sign of coefficient $a_{2}$. Because of the complexity of terms that come into the calculation of $a_{2}$, the analytic determination of its sign is very difficult. However, we can notice that the field at the second order is generated by the passing of $U^{0}$ and $U^{1}$ (the latter being generated by $U^{0}$ ). So, in accordance with the principle of causality, the component of $U^{2}$ following $D^{a}$, which is in quadrature of phase, can only be phase late. We can then deduce that: $a_{2}>0$ and $V_{a}^{\prime}<V_{a}$, i.e. $V_{a}^{\prime}$ decreasing with the frequency.

We have finally specified that this description was only valid for a distance of propagation limited to $\delta_{p}=O(\lambda / 2 \pi \sqrt{ } \varepsilon)$. We will deal with this point in detail in paragraph 3.3.

3.2.3 Order 3: attenuation. In this paragraph, we deal only with the perturbation brought by the taking into account of the terms of third order. Equation (4.3) shows that the sources come from the three wave fields $U^{0}, U^{1}$, and $U^{2}$. Let's first calculate these volume forces.

The sources resulting from $U^{0}$, are given by:

$$
-S^{3}\left(U^{0}\right)=-\left[\nabla . C^{3} \ldots . . \nabla \nabla \nabla e\left(U^{0}\right)+\omega^{2} R^{3} \ldots \nabla \nabla e\left(U^{0}\right)\right]
$$

Thus in the frame $E_{p}$ :

$$
-S_{i}^{3}\left(U^{0}\right)=-\left[C_{p i j}^{3 k l m n u} U_{u, n m l k j}^{0}+\omega^{2} R_{p i}^{3 q r s t} U_{t, s r q}^{0}\right]
$$

which gives in a matricial form and when introducing the expression (5) of $U^{0}$ :

where:

$$
-S^{3}\left(U^{0}\right)=-\left(-i k_{a}\right)^{3} Q_{p}^{3} D^{a} \exp \left(-i k_{a} x\right)
$$

$$
Q_{p i j}^{3}=\left(-i k_{a}\right)^{2} C_{p i 1}^{3111 j}+\omega^{2} R_{p i}^{311 j}
$$

As for the source resulting from $U^{1}$, we can easily get with the expression (6) of $U^{1}$ :

$$
-S^{2}\left(U^{1}\right)=\left(i k_{a}\right)^{3} Q_{p}^{2}\left(b_{1} D^{b}+c_{1} D^{c}\right) \exp \left(-i k_{a} x\right)
$$

Finally, the volume forces resulting from $U^{2}$ must be split in two parts: On the one hand, the terms that result from the components of $U^{2}$ polarized according to $D^{b}$ and $D^{c}$ :

$$
\left(-i k_{a}\right)^{3} Q_{p}^{1}\left(b_{2} D^{b}+c_{2} D^{c}\right) \exp \left(-i k_{a} x\right)
$$

On the other hand, the terms that result from the amplified component following $D^{a}$ :

with:

$$
-S^{1}\left[\left(-i k_{a} x\right) a_{2} D^{a}\left(k_{a}\right)^{2} \exp \left(-i k_{a} x\right)\right]=\left(-i k_{a}\right)^{3}\left[\left(-i k_{a} x\right) Q_{p}^{1}+Q_{p}^{\prime 1}\right] a_{2} D^{a} \exp \left(-i k_{a} x\right)
$$

$$
Q_{p i j}^{\prime 1}=3\left(-i k_{a}\right)^{2} C_{p i 1}^{111 j}+\omega^{2} R_{p i}^{11 j}
$$

Finally we obtain for the set of sources:

$$
\left[Q_{p}^{3} D^{a}-Q_{p}^{2}\left(b_{1} D^{b}+c_{1} D^{c}\right)+Q_{p}^{1}\left(a_{2} D^{a}\left(-i k_{a} x\right)+b_{2} D^{b}+c_{2} D^{c}\right)+Q_{p}^{\prime 1} a_{2} D^{a}\right]\left(-i k_{a}\right)^{3} \exp \left(-i k_{a} x\right)
$$

The geometry of this distribution of sources leads to a wave field of the following form:

$$
U^{3}=\left[\left(-i k_{a} x\right) a_{3} D^{a}+b_{3}\left(1+\beta\left(i k_{a} x\right)\right) D^{b}+c_{3}\left(1+\gamma\left(i k_{a} x\right)\right) D^{c}\right]\left(i k_{a}\right)^{3} \exp \left(-i k_{a} x\right)
$$

(we notice that there is no "double" amplification according to polarization $D^{a}$, because, $D^{a} \cdot Q_{p}^{1} . D^{a}=0$, as has been seen in 3.2).

The determination of the coefficients is done in the same way as precedingly, that is to say by identification of $S^{0}\left(U^{3}\right)$ with the source terms. As seen above it can be shown that the terms of type $\varepsilon^{3} k_{a}^{3} a_{3}$ are real and are really $O\left(\varepsilon^{3}\right)$ whereas $\beta$ and $\gamma$ are $O(1)$.

We observe that the terms polarized in the three directions $D^{a}, D^{b}$ and $D^{c}$, increase linearily with the distance. Moreover the component $D^{a}$ of the diffracted field is in phase opposition (or in phase if $a_{3}<0$ ) with the wave at order zero (see Fig. 6). Consequently the global wave amplitude in the polarization direction $D^{a}$ will be given by the corresponding component $\alpha^{\prime}$ of:

$$
U(x)=U^{0}(x)+\varepsilon U^{1}(x)+\varepsilon^{2} U^{2}(x)+\varepsilon^{3} U^{3}(x)
$$




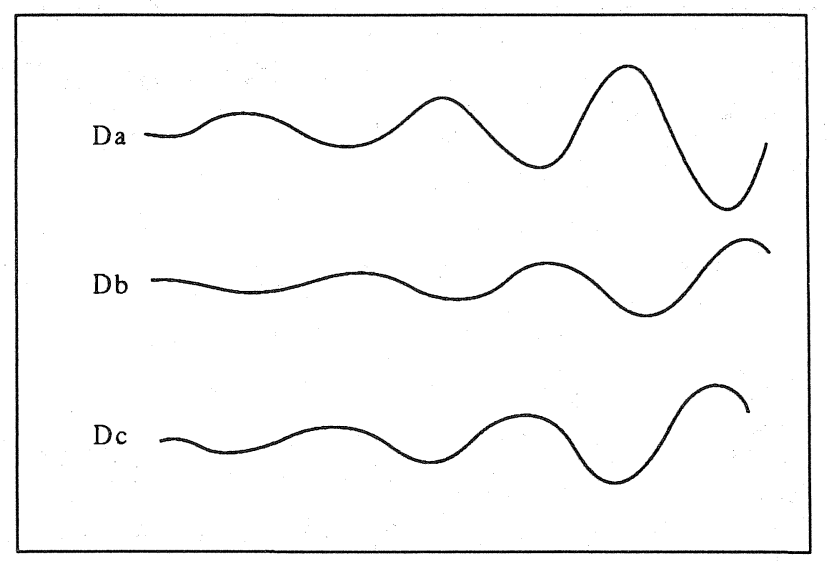

Fig. 6. Wave field at the third order: the vibration for each polarization direction is amplified.

so, when returning to the real expression:

$$
\alpha^{\prime}=\left[1-\varepsilon^{3} a_{3} k_{a}^{3}\left(k_{a} x\right)\right] \cos \left(k_{a} x\right)-\varepsilon^{2} a_{2} k_{a}^{2}\left(k_{a} x\right) \sin \left(k_{a} x\right)
$$

Let's introduce the phase shift $\varphi^{\prime}$ such as:

$$
\begin{gathered}
\operatorname{tg}\left(\varphi^{\prime}\right)=\varepsilon^{2} a_{2} k_{a}^{2}\left(k_{a} x\right)\left[1-\varepsilon^{3} a_{3} k_{a}^{3}\left(k_{a} x\right)\right]^{-1} \\
\alpha^{\prime}=\left(1-\varepsilon^{3} a_{3} k_{a}^{3}\left(k_{a} x\right)\right) \cos \left(k_{a} x+\varphi^{\prime}\right)\left[\cos \left(\varphi^{\prime}\right)\right]^{-1}
\end{gathered}
$$

But we have seen that $a_{2} k_{a}^{2}$ and $a_{3} k_{a}^{3}$ are $O(1)$. Consequently, for a distance $x$ inferior to $\delta_{p}$ :

Thus:

$$
\begin{gathered}
0<k_{a} x<\varepsilon^{-1 / 2} \quad \varphi^{\prime}=O\left(\varepsilon^{3 / 2}\right) \\
\operatorname{tg}\left(\varphi^{\prime}\right)=\varphi^{\prime}\left(1+O\left(\varepsilon^{3}\right)\right) \quad \cos \left(\varphi^{\prime}\right)=1-1 / 2 \varphi^{\prime 2}+O\left(\varepsilon^{6}\right)
\end{gathered}
$$

$$
\alpha^{\prime}=\left(1-\varepsilon^{3} a_{3} k_{a}^{3}\left(k_{a} x\right)\right)\left(1+\varepsilon^{4}\left(a_{2} k_{a}^{2}\left(k_{a} x\right)\right)^{2} / 2\right) \cos \left(k_{a} x+\varphi^{\prime}\right)
$$

so, to $\varepsilon^{4}$ (for both amplitude and phase):

$$
\alpha^{\prime}=\left[1-\varepsilon^{3} a_{3} k_{a}^{3}\left(k_{a} x\right)\right] \cos \left[k_{a}\left(1+\varepsilon^{2} a_{2} k_{a}^{2}\right) x\right]
$$

We therefore notice the same correction on celerity as at the second order, but we see that the amplitude varies linearily during the propagation. The direction of variation is given by the sign of $a_{3}$ whose analytic determination is extremely complicated. However, when assuming that during the propagation, the energy cannot increase at the macroscopic level, we are led to consider that $a_{3}>0$, which corresponds to a decreasing of amplitude.

Although this attenuation only appears at the third order, it is the most important phenomenon in the experimental field because it is the easiest to observe. Such an attenuation of the wave results from destructive interferences between the wave of zero order and the diffracted wave of order three (see Fig. 7). Since $k_{a}^{3} a_{3}=O(1)$, the attenuation on a distance of the size of period $l=\varepsilon L=\varepsilon / k_{a}$ is equal to:

$$
\zeta_{l}=\varepsilon^{3} k_{a}^{3} a_{3}\left(k_{a} \varepsilon / k_{a}\right)=\varepsilon^{4} k_{a}^{3} a_{3}=O\left(\varepsilon^{4}\right)=O\left(\left(\omega l / V_{a}\right)^{4}\right)
$$

We will have for one unit length:

$$
\zeta=O\left(l^{3}\left(\omega / V_{a}\right)^{4}\right)=O\left(l^{3}\left(k_{a}\right)^{4}\right)
$$

And then for one wave length:

$$
\zeta_{\lambda}=O\left(2 \pi l^{3}\left(\omega / V_{a}\right)^{3}\right)=O\left(2 \pi l^{3}\left(k_{a}\right)^{3}\right)
$$

Thus, we are back to the usual expressions for the attenuation of waves by Rayleigh diffraction in a heterogeneous medium, but the results have been established here for very heterogeneous 


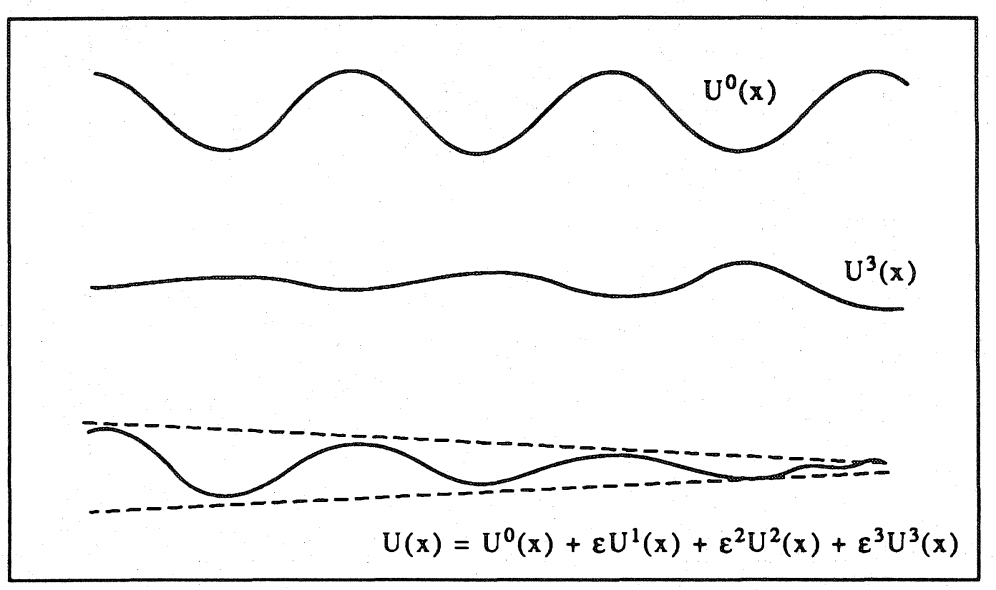

Fig. 7. The effect of attenuation results of the summation of the wave $U^{0}$ and the wave $U^{3}$ (in phase opposition and amplified).

materials (as far as the constrast of the mechanical properties and the concentration of inclusions are concerned).

Here again, the description given is only correct for distances inferior to $\delta_{p}$ (at such a distance the attenuation of the wave is of the order of $\left.O\left(\varepsilon^{5 / 2}\right)\right)$. The analysis of the wave beyond that distance is dealt with in 3.3 .

3.2.4 Superior orders. The calculation of superior terms becomes very complex even in the case of a plane wave. However as these terms can only generate (at superior orders) phenomena of the same nature as those that have already been put in evidence, it is not necessary to develop them.

3.2.5 Case of macroscopically isotropic media. As far as strictly isotropic materials are concerned-that is to say isotropic at each order-we have seen that the operators of odd rank are equal to zero. In such conditions it is obvious that there are no sources at the first order and that the field is identically equal to zero. At the second order, there still is the distribution of volume forces $-S^{2}\left(U^{0}\right)$. $S^{2}$ being an isotropic operator, these forces are oriented in the direction of polarization of the wave considered $(P$ or $S)$. Therefore they generate a diffracted field $U^{2}$ that is also polarized in this direction:

$$
U^{2}=\left(-i k_{a} x\right) a_{2} D^{a}\left(k_{a}\right)^{2} \exp \left(-i k_{a} x\right)
$$

We can therefore deduce that at the second order the global wave stays polarized in the same direction but shows a dispersion of celerity. It is easy to check that the sources of third order are equal to zero and that therefore there is no diffracted field at this order. When reasoning by recurrence, we can check that all the fields of odd order are equal to zero and that the fields of even order all have the same polarization and oscillate in quadrature of phase with the wave of zero order. Finally, in a heterogeneous medium, which is strictly isotropic at the macroscopic level, the only effect of the scattering is the dispersion of celerity. (As a matter of fact, as we have already mentioned in paragraph 2.2.4, the case of a strict isotropy seems difficult to achieve in periodical heterogeneous media but materials can be isotropic up to a certain order.)

\subsection{Evolution of the wave during its propagation}

3.3.1 Limit of validity of the macroscopic description. From the preceding study, we can see that the description of the propagation of macroscopic waves is valid in any point of the infinite heterogeneous medium if we only take terms of order zero or one into account. On the contrary the analysis of the effects of the scattering at superior orders is only correct on a distance of propagation $\delta_{p}$ which is large but limited in comparison with the wave length.

Such a restriction of the domain of analysis comes from the amplification phenomenon which 


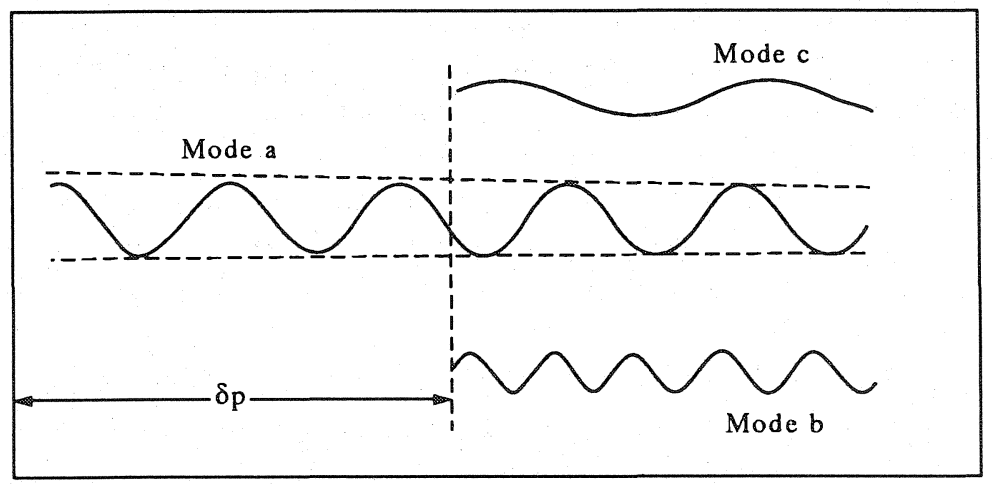

Fig. 8. From the distance $\delta p$, mode conversion appears at the second order.

generates wave fields increasing with the distance of propagation. As a result, beyond the distance $\delta_{p}$ the terms of order $i$ change of range and interact with terms of order $i-1$. Since the fundamental hypothesis of discrimination of different orders is not respected any longer, the results of the homogenization are not correct any longer.

3.3.2 Description of the wave field beyond the distance $\delta_{p}=(c / \omega)^{3 / 2} \vee l$. To avoid this difficulty we follow this reasoning. In the vicinity of distance $\delta_{p}$, in a plane parallel to the wave front, the medium is not just solicited by the displacements of the attenuated wave at order zero any longer. Moreover, there appear displacements of order one polarized according to $D^{a}$ (resulting from the amplified wave of order 2) and displacements of order 2 polarized in the three directions (resulting from amplified waves of order 3)... In order to satisfy these new boundary conditions a new wave of order one, polarized according to $D^{a}$, and propagating in the same direction as the wave of order zero is added to it, as well as waves of order 2 polarized according to the three directions etc... For each of these waves, the analysis done earlier is still applicable and the global wave field can be described theoretically.

Let's particularly mention that from the distance $\delta_{p}$, we can observe a conversion of mode at order 2, that is to say that the displacement polarized according to $D^{b}$ and $D^{c}$, of order 2, will propagate at $V_{b}$ and $V_{c}$ celerities (see Fig. 8). So we will start to notice in the wave motion, movements of weak amplitude that propagate at different celerities from the one of the initial wave.

3.3.3 Mode conversions. It is obvious that the more the wave progresses, the more the diffracted wave motion which is associated to it becomes complex. The converted modes become more and more important. As the "resonant" waves are linearily amplified, at a distance of:

$$
\varepsilon^{-1} \delta_{p}=\lambda / \varepsilon^{3 / 2} 2 \pi=l^{3 / 2}\left(V_{a} / \omega\right)^{5 / 2}
$$

the mode conversion appears at the first order. Finally, beyond a distance of:

$$
\varepsilon^{-2} \delta_{p}=\lambda / \varepsilon^{5 / 2} 2 \pi=l^{5 / 2}\left(V_{a} / \omega\right)^{7 / 2}
$$

the diffracted terms interfere with the wave at order zero so that the amplitude of the global wave decreases to order one. So, from this characteristic distance, the global wave is attenuated by a factor $\varepsilon$, and the wave field is constituted of the three modes.

3.3.4 Case of macroscopically isotropic materials. For these materials the fields at each order are polarized in the same direction. So there is no mode conversion and the description does not change beyond the distance $\delta_{p}$.

\section{CONCLUSION}

We have used the homogenization method to study the propagation of waves in elastic media with a periodic microstructure when the wave length is large in comparison with the dimension 
of the heterogeneities. We have shown that when we only consider the zero order, the macroscopic description is identical to the one we would obtain for a homogeneous material. Taking into account terms of a superior order we are led apart from the classic behaviour of homogeneous media. The perturbations directly come from the heterogeneous nature of the material and are easily interpretable in the context of the wave propagation. Actually, we are back to the fact that when a wave passes through heterogeneities it generates a distribution of source that produces a new wave field etc... This is therefore the experimentally well known phenomenon of scattering.

For a harmonic plane wave of celerity $V_{a}$, the analysis of the effects of the corrective terms allows to put in evidence a correction of polarization at the first order (that varies in $\omega l / V_{a}$ ), and then at the second order a celerity dispersion (varying according to $\left.\left(\omega l / V_{a}\right)^{2}\right)$ and finally at the third order a wave attenuation by length unit (of order $\left(\omega l / V_{a}\right)^{4} / l$ ). Those last two phenomena result from constructive interferences (order 2) or destructive (order 3 ) between the initial wave of order zero and the waves generated by the scattering. On the other hand, this description is only correct on a distance of propagation $\delta_{p}=O\left(\left(V_{a} / \omega\right)^{3 / 2} \sqrt{ } l\right)$ which is large in comparison with the wave length, but limited. Actually, from such a distance, mode conversions at the second order appear. These converted modes change to order one from a distance $O\left(l^{3 / 2}\left(V_{a} / \omega\right)^{5 / 2}\right)$, and after a travel distance $O\left(l^{5 / 2}\left(V_{a} / \omega\right)^{7 / 2}\right)$, the global wave amplitude decreases to order one and is then formed of three modes. Different ranges of magnitude of these quantities are presented in Table 1 , for three cases of dynamic excitations in "geomaterials":

-propagation of seismic waves in alluvium;

- geophysical survey in sandstone;

-ultrasonic tests in cellular concrete.

We have also shown that the deviation of polarization, the attenuation of the wave and the mode conversions are directly associated to the macroscopic anisotropy of the medium. So as far as macroscopically isotropic heterogeneous materials are concerned the dispersion of celerity is the only one to remain.

Thus, the homogenization method developed to the third order allows to describe very accurately the Rayleigh diffraction. Moreover, such a method gives a theoretical means to quantify these effects from the knowledge of the mechanical properties of the period. The calculation of the different tensors that come into the macroscopic description can be made through the solving (using finite elements or integral equations), of three types of problems set on the period. However the numerical resolution is complex because of the high number of terms to calculate but also because the solutions at order three are determined from solutions at order two, which themselves depend on solutions at order one. This therefore requires a very high quality in the numerical resolution.

Let's notice that we can use such an approach for materials that show:

- heterogeneities with important contrasts in properties or that have a clearly anisotropic behaviour (which makes the method of perturbations of elastic coefficients; i.e. Born approximation-inapplicable)

Table 1. Examples of applications for three geomaterials

\begin{tabular}{|c|c|c|c|c|c|c|c|c|}
\hline & $l(\mathrm{~mm})$ & $C(\mathrm{~m} / \mathrm{s})$ & $F(\varepsilon=1)(\mathrm{Hz})$ & $f(\mathrm{~Hz})$ & $\lambda$ & $\varepsilon$ & $0(\zeta)\left(\mathrm{m}^{-1}\right)$ & $0\left(\delta_{p}\right)$ \\
\hline Alluvium & $50 \mathrm{~mm}$ & $\begin{array}{l}C_{p}=500 \mathrm{~m} / \mathrm{s} \\
C_{s}=170 \mathrm{~m} / \mathrm{s}\end{array}$ & $\begin{array}{c}1.6 \mathrm{kHz} \\
550 \mathrm{~Hz}\end{array}$ & $\begin{array}{c}\text { Seismic } \\
5 \mathrm{~Hz}\end{array}$ & $\begin{array}{r}100 \mathrm{~m} \\
34 \mathrm{~m}\end{array}$ & $\begin{array}{l}0.003 \\
0.009\end{array}$ & $\begin{array}{c}\sim 10^{-9} \\
1.5 \times 10^{-6}\end{array}$ & $\begin{array}{l}1.8 \mathrm{~km} \\
360 \mathrm{~m}\end{array}$ \\
\hline Sandstone & $1 \mathrm{~mm}$ & $\begin{aligned} C_{p}^{s} & =1250 \mathrm{~m} / \mathrm{s} \\
C_{s} & =750 \mathrm{~m} / \mathrm{s}\end{aligned}$ & $\begin{array}{l}200 \mathrm{kHz} \\
120 \mathrm{kHz}\end{array}$ & $\begin{array}{c}\text { Geophysics } \\
5 \mathrm{kHz}\end{array}$ & $\begin{array}{l}0.25 \mathrm{~m} \\
0.15 \mathrm{~m}\end{array}$ & $\begin{array}{l}0.025 \\
0.04\end{array}$ & $\begin{array}{l}4 \times 10^{-4} \\
3 \times 10^{-3}\end{array}$ & $\begin{array}{c}1.6 \mathrm{~m} \\
0.75 \mathrm{~m}\end{array}$ \\
\hline Cellular concrete & $2 \mathrm{~mm}$ & $C_{p}=2000 \mathrm{~m} / \mathrm{s}$ & $160 \mathrm{kHz}$ & $\begin{array}{l}\text { Ultrasound } \\
50 \mathrm{kHz}\end{array}$ & $40 \mathrm{~mm}$ & 0.3 & 5 & $70 \mathrm{~mm}$ \\
\hline
\end{tabular}


-a finite concentration of heterogeneities-so that the "scattering diffraction points" are close one to the other and that the hypothesis of the far field is not verified.

Let's finally remind the readers that these results have been established when assuming that the medium was microperiodical and that $\varepsilon \ll 1$ :

-If the hypothesis of periodicity is not verified the method is not directly applicable any longer. However, in many problems, the structure of the equations of a homogenizable medium is identical, whether the microstructure of the medium is periodic or stochastic [Auriault, 1991]. This seems to be proved by the fact that descriptions that have been obtained are in agreement with experimental results concerning metals or fissured rocks whose structure is not periodical.

-As far as $\varepsilon$ is concerned, we generally admit that the theory remains applicable as long as $\varepsilon<0.1$. (when using Fourier transform, we can propagate transitory signals whose spectra is limited by this condition). For higher values, the physic of waves becomes much more complex because the different orders interfere. The role of microstructure becomes important, and periodical or stochastic media have then a very different behaviour. It is then not possible any longer to obtain a macroscopic description.

Acknowledgement-This research was supported by the GRECO "Geomateriaux" of the French Centre National de la Recherche Scientifique.

\section{REFERENCES}

[1] J. L. AURIAULT, Int. J. Engng Sci. 29 (7), 785-795 (1991).

[2] J. L. AURIAULT and G. BONNET, Arch. Mech. 37 (4-5), 269-284 (1985).

[3] A. BENSOUSSAN, J. L. LIONS and G. PAPANICOLAOU, Asymptotic Analysis for Periodic Structures. North-Holland, Amsterdam, (1978).

[4] C. BOUTIN, Diffraction Rayleigh dans les milieux élastiques hétérogénes CNRS, GRECO Geomateriaux Aussois. (November 1991).

[5] C. BOUTIN and J. L. AURIAULT, Int. J. Engng Sci. 28 (11), 1157-1181 (1990).

[6] L. J. BOND, Numerical techniques and their use to study wave propagation and scattering-A review. Elastic Wave and Ultrasonic Nondestructive Evaluation, pp. 17-27. Proc of IUTAM. Boulder, CO (1989).

[7] B. GAMBIN and E. KRÖNER, Phys. Stat. Sol. (b) 151, 513-519 (1989).

[8] J. E. GUBERNATIS, E. DOMANY, J. A. KRUMHANSL and M. HUBERNAM, J. Appl. Phys. 48, (1977).

[9] S. HIRSEKORN, J. Acoust. Soc. Am. 83 (4) (1988).

[10] A. LASZLO, J. ROSE and C. MOBLY, J. Appl. Phys. 59 (2) (1986).

[11] M. PIAU, Int. J. Engng Sci. 18, 549-568 (1980).

[12] E. SANCHEZ PALENCIA, Non Homogeneous Media and Vibration Theory. Lecture Notes in Physics, 127. Springer. Berlin (1980).

[13] F. STANKE and G. KINO, J. Acoust. Soc. Am. 75 (3), 665-681 (1984).

[14] N. TURBE, J. Math. Meth. Appl. Sci. (3), 433-449 (1982).

(Received 4 April 1992; accepted 21 December 1992)

\section{APPENDIX 1}

Homogenization of Elastic Periodic Microstructures

In this Appendix we give the theoretical developments which lead us to the macroscopic balance equations up to the third order. The problems to be solved at the different orders can be treated in the same manner. Therefore we will not go into the mathematical details which can be found in [B, 1978] or [S, 1980].

\section{A1.1 General formulation of the different problems}

Let us mention that all the problems are systematically of the following type. The forcing terms $F$ and $V$ being known and $\Omega$-periodic, we have to find a $\Omega$-periodic continuous displacement field $u$, such as:

$$
\nabla_{y} . \sigma=F \quad \sigma=c(y) . .\left(e_{y}(u)+V\right)
$$

This problem can be put in a variational form by using the following identity, which is valid for any continuous field $w$ :

$$
\int_{\Omega} w \cdot \nabla_{y} \cdot \sigma \mathrm{d} \Omega=-\int_{\Omega} \nabla_{y}(w) . . \sigma \mathrm{d} \Omega+\int_{\partial \Omega} \sigma . n \cdot w \mathrm{~d} s
$$

ES $31: 12-H$ 
By using $\Omega$-periodic test fields $w$ (which allows to suppress the last integral), and because of the symmetry of the stress tensor, we obtain:

$$
\int_{\Omega} e_{y}(w) . . c(y) . . e_{y}(u) \mathrm{d} \Omega=-\int_{\Omega} w . F \mathrm{~d} \Omega-\int_{\Omega} e_{y}(w) . . c(y) . . V \mathrm{~d} \Omega
$$

The properties of the elastic tensor $c$ allow to apply the Lax-Milgramm lemma, which ensures us of the existence of $u$. It is clear that the solution is only determined up to a constant on the cell. The uniqueness is therefore obtained by adding to the problem the condition of zero average. In order to simplify the writing we will write $c$ and $\rho$ instead of $c(y)$ and $\rho(y)$.

\section{A1.2 Resolution of the problems at different orders}

Order $\varepsilon^{-2}$. The first problem to solve is the following (2.2):

$$
L^{-2}\left(U^{0}\right)=\nabla_{y} \cdot c . . e_{y}\left(U^{0}\right)=0
$$

whose evident solutions are constant fields on the period: $U^{0}=U^{0}(x)$

Order $\varepsilon^{-1}$. At this order we are led to the system (2.1):

$$
L^{-2}\left(U^{1}\right)=-\left[L^{-1}\left(U^{(0)}\right)\right]
$$

which, taking into account the expression of $U^{0}$, can be written:

$$
\nabla_{y} . c . .\left(e_{y}\left(U^{1}\right)+e_{x}\left(\overline{U^{0}}\right)\right)=0
$$

As a consequence of the linearity of the problem, the general solution is:

$$
U^{1}(x, y)=X^{1}(y) . . e_{x}\left(\overline{U^{0}(x)}\right)+\overline{U^{1}}(x)
$$

The third rank tensor $X^{1}$ is constructed from the particular solutions $X^{1 k l}$ such as:

$$
C^{0}(y)=c . . e_{y}\left(X^{1}\right)+c \quad \nabla_{y} . C^{0}(y)=0 \quad \overline{X^{1}}=0
$$

(with the convention that $e_{y}(-)$ or $e_{x}(-)$ represents the symmetrized gradient tensors). The corresponding variational formulation is the following:

$$
\int_{\Omega} C^{0}(y) . . e_{y}(w) \mathrm{d} \Omega=\int_{\Omega}\left[e_{y}(w) . . c . . e_{y}\left(X^{1}\right)+c . . e_{y}(w)\right] \mathrm{d} \Omega=0
$$

Using index notations, we have more explicitly:

$$
U_{i}^{1}=X_{i}^{1 k l} e_{x k l}\left(\overline{U^{0}}\right)+\overline{U_{i}^{1}}
$$

The vectors $X^{1 k l}$ being the solutions to the systems:

$$
C_{i j}^{0 k l}=c_{i j}^{k l}+c_{i j}^{s t} e_{y s t}\left(X^{1 k l}\right) \quad\left(C_{i j}^{0 k l}\right)_{, j}=0 \quad \overline{X_{i}^{1 k l}}=0
$$

$\operatorname{Order} \varepsilon^{0}$, From this order the macroscopic balance equations are not obvious any longer. We obtain them by integrating on the cell the considered system (2.0):

$$
L^{-2}\left(U^{2}\right)=-\left[L^{-1}\left(U^{1}\right)+L^{0}\left(U^{0}\right)\right]
$$

wich is more convenient to write in its equivalent form:

$$
\nabla_{y} . \sigma^{1}+\nabla_{x} . \sigma^{0}+\omega^{2} \rho U^{0}=0 \quad \sigma^{i}=c(y) . .\left(e_{y}\left(U^{i+1}\right)+e_{x}\left(U^{i}\right)\right)
$$

Taking into account the stress periodicity, we have:

$$
\nabla_{x} . \overline{\sigma^{0}}+\omega^{2} \bar{\rho} \overline{U^{0}}=0
$$

Then, putting the expression of $U^{1}$ in $\sigma^{0}$, we deduce the macroscopic momentum equation at zero order (3.0):

$$
\nabla_{x} \cdot \overline{C^{0}} . . e_{x}\left(\overline{U^{0}}\right)+\omega^{2} \bar{\rho} \overline{U^{0}}=0
$$

The set of equations allowing the determination of $U^{2}$ is:

$$
\nabla_{y} . c . .\left(e_{y}\left(U^{2}\right)+e_{x}\left(U^{1}\right)\right)=-\left[\nabla_{x} . c . .\left(e_{y}\left(U^{1}\right)+e_{x}\left(U^{0}\right)\right)+\omega^{2} \rho U^{0}\right]
$$

In this equation we substicute $U^{1}$ by its expression and $U^{0}$ by using the macroscopic dynamics equation (3.0) obtained above. We get:

$$
\nabla_{y} . c . .\left(e_{y}\left(U^{2}\right)+e_{x}\left(X^{1} . . e_{x}\left(\overline{U^{0}}\right)\right)+\nabla_{y} . c . . e_{x}\left(\overline{U^{1}}\right)=-\nabla_{x} .\left[C^{0} . . e_{x}\left(\overline{U^{0}}\right)-\beta \overline{C^{0}} . . e_{x}\left(\overline{U^{0}}\right)\right]\right.
$$

In order to simplify the writing, we have introduced $\beta(y)$, the ratio of the density to the average density:

$$
\beta(y)=\rho(y)[\bar{\rho}]^{-1}
$$

We observe that the solution $U^{2}$ depends on two forcing terms:

- The first one is associated to the deformation tensor of the average displacement at the first order,

- The second one is associated to the gradient of the deformation tensor of the average displacement at order zero.

As a consequence of the linearity of the system, the field solution is a linear combination of particular solutions associated to each of these forcing terms. It is important to notice that the 
problems linked to the displacement at the first order are identical to those already treated at order zero. Consequently we have:

$$
U^{2}(x, y)=X^{2}(y) \ldots \nabla_{x} e_{x}\left(\overline{U^{0}(x)}\right)+X^{1}(y) . . e_{x}\left(\overline{U^{1}(x)}\right)+\overline{U^{2}}(x)
$$

In which the fourth rank tensor $X^{2}$ is constructed from the particular solutions $X^{2 k l m}$ and verifies:

$$
C^{1}(y)=c . . e_{y}\left(X^{2}\right)+c . X^{1} \quad \nabla_{y} \cdot C^{1}(y)=-\left[C^{0}-\beta \overline{C^{0}}\right] \quad \overline{X^{2}}=0
$$

Which corresponds to the variational formulation:

$$
\int_{\Omega} C^{1}(y) . . e_{y}(w) \mathrm{d} \Omega=\int_{\Omega}\left[e_{y}(w) . . c . . e_{y}\left(X^{2}\right)+c . X^{1} . . e_{y}(w)\right] \mathrm{d} \Omega=\int_{\Omega}\left[C^{0}(y)-\beta(y) \overline{C^{0}}\right] . w \mathrm{~d} \Omega
$$

Or using the indicial notation:

$$
U_{i}^{2}=X_{i}^{2 k l m} \nabla_{x} e_{x}\left(\overline{U^{0}}\right)_{k l m}+X_{i}^{l p q} e_{x p q}\left(\overline{U^{1}}\right)+\overline{U_{i}^{2}}
$$

The vectors $X^{2 k l m}$ being the solutions to the systems:

$$
C_{i j}^{1 k l m}=C_{i j}^{m r} X_{r}^{1 k l}+c_{i j}^{s t} e_{y s t}\left(X^{2 k l m}\right) \quad\left(C_{i j}^{1 k l m}\right)_{, j}=\beta \overline{C_{i m}^{0 k l}}-C_{i m}^{0 k l} \quad \overline{X_{i}^{2 k l m}}=0
$$

$\operatorname{Order} \varepsilon^{1}$. As above, following the same method, we first establish the balance equation at this order. We simply obtain:

$$
\nabla_{x} \cdot \overline{\sigma^{1}}+\omega^{2} \overline{\rho U^{1}}=0 \quad \overline{\sigma^{1}}=\overline{c . .\left(e_{y}\left(U^{2}\right)+e_{x}\left(U^{1}\right)\right)}
$$

In order to have an equation where only the average displacements appear, we introduce the expressions of the fields that have already been determined at the first and second orders. Thus, we get:

$$
\begin{aligned}
\sigma^{1} & =c . .\left[e_{y}\left(X^{2} \ldots \nabla_{x} e_{x}\left(\overline{U^{0}}\right)+X^{1} . . e_{x}\left(\overline{U^{1}}\right)\right)+e_{x}\left(X^{1} . . e_{x}\left(\overline{U^{0}}\right)+\overline{U^{1}}\right)\right] \\
& =C^{1} . . \nabla_{x} e_{x}\left(\overline{U^{0}}\right)+C^{0} . . e_{x}\left(\overline{U^{1}}\right)
\end{aligned}
$$

Consequently, the momentum balance at the first order is (3.1):

$$
\nabla_{x} \cdot \overline{C^{0}} . . e_{x}\left(\overline{U^{1}}\right)+\omega^{2} \bar{\rho} \overline{U^{1}}=-\left[\nabla_{x} \cdot C^{1} \ldots \nabla_{x} e_{x}\left(\overline{U^{0}}\right)+\omega^{2} \overline{\rho X^{1}} . . e_{x}\left(\overline{U^{0}}\right)\right]
$$

The determination of the field $U^{3}$, is achieved by solving:

$$
\nabla_{y} . c . .\left(e_{y}\left(U^{3}\right)+e_{x}\left(U^{2}\right)\right)=-\left[\nabla_{x} . c . .\left(e_{y}\left(U^{2}\right)+e_{x}\left(U^{1}\right)\right)+\omega^{2} \rho U^{1}\right]
$$

That is, when expressing the different fields:

$$
\begin{aligned}
\nabla_{y} . c . .\left[e_{y}\left(U^{3}\right)+e_{x}\left(X^{2} \ldots \nabla_{x} e_{x}\left(\overline{U^{0}}\right)\right]+\nabla_{y} . e_{x}\left(X^{1} . . e_{x}\left(\overline{U^{1}}\right)\right)+\nabla_{y} \cdot e_{x}\left(\overline{U^{2}}\right)\right. \\
=-\nabla_{x} \cdot c . .\left[e _ { y } \left(X^{2} \ldots \nabla_{x} e_{x}\left(\overline{U^{0}}\right)+e_{x}\left(X^{1} . . e_{x}\left(\overline{U^{0}}\right)\right]\right.\right. \\
\quad-\nabla_{x} \cdot c . .\left[e_{y}\left(X^{1} . . e_{x}\left(\overline{U^{1}}\right)+e_{x}\left(\overline{U^{1}}\right)\right]-\omega^{2} \rho\left[X^{1} . . e_{x}\left(\overline{U^{0}}\right)+\overline{U^{1}}\right]\right.
\end{aligned}
$$

Let us now replace the average displacement at the first order by using the momentum balance (3.1):

$$
-\omega^{2} \rho \overline{U^{1}}=\beta\left[\nabla_{x} . C^{0} . . e_{x}\left(\overline{U^{1}}\right)+\nabla_{x} . C^{1} \ldots \nabla_{x} e_{x}\left(\overline{U^{0}}\right)+\omega^{2} \overline{\rho X^{1}} . . e_{x}\left(\overline{U^{0}}\right)\right]
$$

Moreover, with the dynamics equation (3.0), we have:

$$
\omega^{2} \bar{\rho} e_{x}\left(\overline{U^{0}}\right)=-e_{x}\left(\nabla_{x} \cdot \overline{C^{0}} . . e_{x}\left(\overline{U^{0}}\right)\right)
$$

Which finally gives the system:

$$
\begin{array}{r}
\nabla_{y} . c . .\left[e_{y}\left(U^{3}\right)+e_{x}\left(X^{2} \ldots \nabla_{x} e_{x}\left(\overline{U^{0}}\right)\right]+\nabla_{y} \cdot e_{x}\left(X^{1} . . e_{x}\left(\overline{U^{1}}\right)+\nabla_{y} \cdot e_{x}\left(\overline{U^{2}}\right)\right.\right. \\
=-\nabla_{x} \cdot\left[C^{1}-\beta \overline{C^{1}}\right] \ldots \nabla_{x} e_{x}\left(\overline{U^{0}}\right)-\beta\left([\bar{\rho}]^{-1} \overline{\rho X^{1}}-X^{1}\right) . . e_{x}\left(\nabla_{x} \cdot \overline{C^{0}} . . e_{x}\left(\overline{U^{0}}\right)\right)-\nabla_{x} \cdot\left[C^{0}-\beta \overline{C^{0}}\right] \ldots e_{x}\left(\overline{U^{1}}\right)
\end{array}
$$

We notice that the solution $U^{3}$ depends of three forcing terms associated to:

- the deformation tensor of the average displacement at the second order;

- the gradient of deformation tensor of the average displacement at the first order;

- the double gradient of deformation tensor of the displacement at zero order. This latter term only, introduces a new problem to solve. As a matter of fact the first two terms lead to problems already solved at the previous orders.

Because of the linearity the field solution is as follows:

$$
U^{3}(x, y)=X^{3}(y) \ldots \nabla_{x} \nabla_{x} e_{x}\left(\overline{U^{0}(x)}\right)+X^{2}(y) \ldots \nabla_{x} e_{x}\left(\overline{U^{1}(x)}\right)+X^{1}(y) . . e_{x}\left(\overline{U^{2}(x)}\right)+\overline{U^{3}}(x)
$$

$X^{3}$ is the 5 th rank tensor constructed from the particular solutions $X^{3 k l m n}$. It verifies the equations:

$$
\begin{gathered}
C^{2}(y)=c . . e_{y}\left(X^{3}\right)+c \cdot X^{2} \quad \overline{X^{3}}=0 \\
\nabla_{y} . C^{2}(y)=-\left(C^{1}-\beta \overline{C^{1}}\right)-\beta\left([\bar{\rho}]^{-1} \overline{\rho X^{1}}-X^{1}\right) \cdot \overline{C^{0}}
\end{gathered}
$$

Or, with indicial notation:

$$
U_{i}^{3}=X_{i}^{3 k l m n} \nabla_{x} \nabla_{x} e_{x}\left(\overline{U^{0}}\right)_{k l m n}+X_{i}^{2 p q r} \nabla_{x} e_{x}\left(\overline{U^{1}}\right)_{p q r}+X_{i}^{1 s t} e_{x s t}\left(\overline{U^{2}}\right)+\overline{U_{i}^{3}}(x)
$$


Where the vectors $X^{3 k l m n}$ are the solutions to the systems:

$$
\begin{aligned}
C_{i j}^{2 k l m n} & =c_{i j}^{n r} X_{r}^{2 k l m}+c_{i j}^{s t} e_{y s t}\left(X^{3 k l m n}\right) \quad \overline{X_{i}^{3 k l m n}}=0 \\
\left(C_{i j}^{2 k l m n}\right)_{, j} & =\beta \overline{C_{i n}^{1 k l m}}-C_{i n}^{1 k l m}-\beta\left([\bar{\rho}]^{-1} \overline{\rho X_{i}^{1 n t}}-X_{i}^{1 n}\right) \overline{C_{t m}^{0 k l}}
\end{aligned}
$$

$\operatorname{Order} \varepsilon^{2}$. The balance equation is obtained as precedingly:

$$
\nabla_{x} \cdot \overline{\sigma^{2}}+\omega^{2} \overline{\rho U^{2}}=0 \quad \overline{\sigma^{2}}=\overline{c . .\left(e_{y}\left(U^{3}\right)+e_{x}\left(U^{2}\right)\right)}
$$

After replacing $U^{2}$ and $U^{3}$ by their expression, we get (3.2):

$$
\nabla_{x} \cdot \overline{C^{0}} . . e_{x}\left(\overline{U^{2}}\right)+\omega^{2} \bar{\rho} \overline{U^{2}}=-\left[\nabla_{x} \cdot \overline{C^{1}} \ldots \nabla_{x} e_{x}\left(\overline{U^{1}}\right)+\omega^{2} \overline{\rho X^{1}} . . e_{x}\left(\overline{U^{1}}\right)\right]-\left[\nabla_{x} \cdot \overline{C^{2}} \ldots \nabla_{x} \nabla_{x} e_{x}\left(\overline{U^{0}}\right)+\omega^{2} \overline{\rho X^{2}} \ldots \nabla_{x} e_{x}\left(\overline{U^{0}}\right)\right]
$$

The differential systems to be solved in order to determine $U^{4}$ are:

$$
\nabla_{y} . c . .\left(e_{y}\left(U^{4}\right)+e_{x}\left(U^{3}\right)\right)=-\left[\nabla_{x} \cdot c . .\left(e_{y}\left(U^{3}\right)+e_{x}\left(U^{2}\right)\right)+\omega^{2} \rho U^{2}\right]
$$

In order to be more precise as to the nature of the solution, we have to substitute the fields $U^{2}$ and $U^{3}$ by their expression. We thus obtain:

$$
\begin{array}{r}
\nabla_{y} . c . .\left(e_{y}\left(U^{4}\right)+e_{x}\left(U^{3}+\sum_{k=0}^{2} X^{k+1} . .\left(\nabla_{x}\right)^{k} e_{x}\left(\overline{U^{2-k}}\right)\right)\right) \\
=-\left[\nabla_{x} . c . .\left(e_{y}\left(\sum_{k=0}^{2} X^{k+1} . .\left(. \nabla_{k}\right)^{k} e\left(\overline{U^{2-k}}\right)\right)+e_{x}\left(U^{2}+\sum_{k=0}^{1} X^{k+1} . .\left(\nabla_{x}\right)^{k} e_{x}\left(\overline{U^{1-k}}\right)\right)\right)\right] \\
-\left[\omega^{2} \rho\left(U^{2}+\sum_{k=0}^{1} X^{k+1} . .\left(. \nabla_{x}\right)^{k} e_{x}\left(\overline{U^{1-k}}\right)\right)\right]
\end{array}
$$

The last momentum balance allows to replace the average field at the second order. So the second member is transformed into:

$$
\sum_{k=0}^{2} \nabla_{x} .\left(\beta \overline{C^{k}}-C^{k}\right) . .\left(. \nabla_{x}\right)^{k} e_{x}\left(\overline{U^{2-k}}\right)+\sum_{k=1}^{2} \omega^{2}\left(\beta \overline{\rho X^{k}}-\rho X^{k}\right) . .\left(. \nabla_{x}\right)^{k-1} e_{x}\left(\overline{U^{2-k}}\right)
$$

Then, putting in the last term the expressions of the average fields given by the momentum balance at the first two orders, (3.0), (3.1), we obtain the forcing term associated to $U^{0}$ :

$$
\begin{aligned}
\nabla_{x} \cdot\left(\beta \overline{C^{2}}-C^{2}\right) \ldots \nabla_{x} \nabla_{x} e_{x}\left(\overline{U^{0}}\right)-\beta\left([\bar{\rho}]^{-1} \overline{\rho X^{2}}-X^{2}\right) \ldots \nabla_{x} e_{x}\left(\nabla_{x} \cdot \overline{C^{0}} . . e_{x}\left(\overline{U^{0}}\right)\right) \\
+\beta\left([\bar{\rho}]^{-1} \overline{\rho X^{1}}-X^{1}\right) . . e_{x}\left[\nabla_{x} \cdot \overline{C^{1}} \ldots \nabla_{x} e_{x}\left(\overline{U^{0}}\right)-\overline{\rho X^{1}} . . e_{x}\left(\nabla_{x} \cdot C^{0} \ldots e_{x}\left(\overline{U^{0}}\right)\right)[\bar{\rho}]^{-1}\right]
\end{aligned}
$$

Here again, the other terms of the second member, associated to the fields of superior orders, lead to problems already solved. Thus the general solution can be split up into four terms:

$$
U^{4}(x, y)=\overline{U^{4}}(x)+\sum_{k=0}^{3} X^{k+1}(y) . .\left(\nabla_{x}\right)^{k}\left(\overline{U^{3-k}}(x)\right)
$$

The tensor $X^{4}$ of 6 th rank, constructed from the particular solutions $X^{4 k l m n p}$, verifies:

$$
\begin{gathered}
C^{3}(y)=c . . e_{y}\left(X^{4}\right)+c \cdot X^{3} \quad \overline{X^{4}}=0 \\
\nabla_{y} \cdot C^{3}(y)=-\left[C^{2}-\beta \overline{C^{2}}\right]-\beta\left([\bar{\rho}]^{-1} \overline{\rho X^{2}}-X^{2}\right) \cdot \overline{C^{0}}-\beta\left([\bar{\rho}]^{-1} \overline{\rho X^{1}}-X^{1}\right) \cdot\left([\bar{\rho}]^{-1} \overline{\rho X^{1}} \cdot \overline{C^{0}}-\overline{C^{1}}\right)
\end{gathered}
$$

Using indicial notation the vectors $X^{4 k l m n}$ are such that:

$$
\begin{aligned}
& C_{i j}^{3 k l m n p}=c_{i j}^{p r} X_{r}^{3 k l m n}+c_{i j}^{s t} e_{y s t}\left(X^{4 k l m n p}\right) \quad \overline{X_{i}^{4 k l m n p}}=0 \\
&\left(C_{i j}^{3 k l m n p}\right)_{, j}= \beta \overline{C_{i p}^{2 k l m n}}-C_{i p}^{2 k l m n}-\beta\left([\bar{\rho}]^{-1} \overline{\rho X_{i}^{2 n p t}}-X_{i}^{2 n p t}\right) \overline{C_{t m}^{0 k l}} \\
&-\beta\left([\bar{\rho}]^{-1} \frac{1}{\rho X_{i}^{1 p s}}-X_{i}^{1 p s}\right)\left(\overline{\rho X_{s}^{1 n r}}[\bar{\rho}]^{-1} \overline{C_{r m}^{0 k l}}-\overline{C_{s n}^{1 k l m}}\right)
\end{aligned}
$$

$\operatorname{Order} \varepsilon^{3}$. At this order we are only interested in the momentum balance, which is given by:

$$
\nabla_{x} . \overline{\sigma^{3}}+\omega^{2} \overline{\rho U^{3}}=0 \quad \overline{\sigma^{3}}=\overline{c . .\left(e_{y}\left(U^{4}\right)+e_{x}\left(U^{3}\right)\right)}
$$

When replacing the fields by their expression calculated above, we obtain (3.3):

$$
\begin{array}{r}
\nabla_{x} \cdot \overline{C^{0}} . . e_{x}\left(\overline{U^{3}}\right)+\omega^{2} \bar{\rho} \overline{U^{3}}=-\left[\nabla_{x}, \overline{C^{1}} \ldots \nabla_{x} e_{x}\left(\overline{U^{2}}\right)+\omega^{2} \overline{\rho X^{1}} . . e_{x}\left(\overline{U^{2}}\right)\right] \\
-\left[\nabla_{x} \cdot \overline{C^{2}} \ldots . \nabla_{x} \nabla_{x} e_{x}\left(\overline{U^{1}}\right)+\omega^{2} \overline{\rho X^{2}} \ldots \nabla_{x} e_{x}\left(\overline{U^{1}}\right)\right] \\
-\left[\nabla_{x} \cdot \overline{C^{3}} \ldots \ldots \nabla_{x} \nabla_{x} \nabla_{x} e_{x}\left(\overline{U^{0}}\right)+\omega^{2} \overline{\rho X^{3}} \ldots \nabla_{x} \nabla_{x} e_{x}\left(\overline{U^{0}}\right)\right]
\end{array}
$$

REMARK. It is interesting to notice that the macroscopic descriptions and the problems on the cell are much more simple in the case of constant density.

\section{APPENDIX 2}

\section{Features of the Diffracted Sources at the First Order}

In this annex we show that at the first order, the orientation of the diffracted volumic forces is orthogonal to the polarization direction of the wave which generates them. This result is important since for this reason the attenuation effect only appears at the third order. 
A2.1 Relation between tensors $C^{\prime}$ and $\rho X^{\prime}$

From the variational formulations we can link the tensors $C^{1}$ and $\rho X^{1}$. Let us transform the term including $X^{2}$, in the expression of the average value of:

$$
C^{1}(y)=c . . e_{y}\left(X^{2}\right)+c . X^{1} \quad C_{i j}^{1 k l m}=c_{i j}^{m r} X_{r}^{1 k l}+c_{i j}^{s t} e_{y s t}\left(X^{2 k l m}\right)
$$

In order to achieve this, we use the variational formulations associated to the fields $X^{1 k l}$. They express that any continuous periodic field $w$ verifies:

$$
\int_{\Omega} c_{s t}^{i j} e_{y s t}(w) \mathrm{d} \Omega=-\int_{\Omega} e_{y}(w) . . c . . e_{y}\left(X^{1 i j}\right) \mathrm{d} \Omega
$$

When we choose $w=X^{2 k l m}$, taking into account the symmetry of $c$, we obtain:

$$
\int_{\Omega}\left[C_{i j}^{1 k l m}-c_{i j}^{m u} X_{u}^{1 k l}\right] \mathrm{d} \Omega=\int_{\Omega} c_{i j}^{s t} e_{y s t}\left(X^{2 k l m}\right) \mathrm{d} \Omega=-\int_{\Omega} e_{y}\left(X^{2 k l m}\right) . . c . . e_{y}\left(X^{1 i j}\right) \mathrm{d} \Omega
$$

Now, using $X^{1 i j}$ as test field in the variational formulation associated to $X^{2 k l m}$, we get:

$$
\text { So: }
$$

$$
-\int_{\Omega} e_{y}\left(X^{2 k l m}\right) . . c . . e_{y}\left(X^{1 i j}\right) \mathrm{d} \Omega=\int_{\Omega}\left[\left(\nabla_{y} \cdot C^{1 k l m}\right) \cdot X^{1 i j}+c_{s t}^{m u} X_{u}^{1 k l} e_{y s t}\left(X^{1 i j}\right)\right] \mathrm{d} \Omega
$$

$$
\int_{\Omega} C_{i j}^{1 k l m} \mathrm{~d} \Omega=\int_{\Omega}\left(\nabla_{y} \cdot C^{1 k l m}\right) \cdot X^{1 i j} \mathrm{~d} \Omega+\int_{\Omega}\left[c_{s t}^{m u} X_{u}^{1 k l} e_{y s t}\left(X^{1 i j}\right)+c_{i j}^{m u} X_{u}^{1 k l}\right] \mathrm{d} \Omega
$$

That is, when introducing $C^{0}$ :

$$
\int_{\Omega} C_{i j}^{1 k l m} \mathrm{~d} \Omega=\int_{\Omega}\left(\nabla_{y} \cdot C^{1 k l m}\right) \cdot X^{1 i j} \mathrm{~d} \Omega+\int_{\Omega} C_{i j}^{0 m u} X_{u}^{1 k l} \mathrm{~d} \Omega
$$

And, replacing the divergency by its expression, we establish the identity:

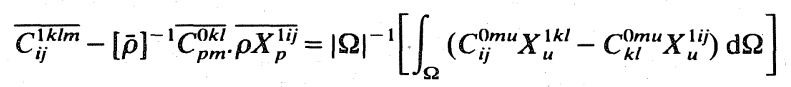

\section{A2.2 Polarization of the sources diffracted at the first order}

We deal here with the orientation of the source vector $S$ whose components are:

$$
S_{i}=\left[\overline{C_{i 1}^{1 k 11}}-[\bar{\rho}]^{-1} \overline{C_{p 1}^{01 k}} \cdot \overline{\rho X_{i}^{11 p}}\right] D_{k}^{a}
$$

Using the above expression of $C^{1}$ :

$$
\overline{C_{i 1}^{1 k 11}}-[\bar{\rho}]^{-1} \overline{C_{p 1}^{01 k}} \cdot \overline{\rho X_{i}^{11 p}}=\overline{C_{i 1}^{01 m} X_{m}^{11 k}-C_{k 1}^{01 m} X_{m}^{11 i}}+[\bar{\rho}]^{-1} \overline{C_{k 1}^{01 p}} \cdot \overline{\rho\left(X_{p}^{1 i 1}-X_{i}^{1 p 1}\right)}
$$

Consequently:

$$
S_{i} \cdot D_{i}^{a}=D_{i}^{a} D_{k}^{a}\left\{\overline{C_{i 1}^{01 m} X_{m}^{11 k}-C_{k 1}^{01 m} X_{m}^{11 i}}+[\bar{\rho}]^{-1} \overline{C_{k 1}^{01 p}} \cdot \overline{\rho\left(X_{p}^{1 i 1}-X_{i}^{1 p 1}\right)}\right\}
$$

The first term of the sum is zero because of its antisymmetry. We transform the second one by using the symmetry of $C^{0}$ and because of the fact that $D^{a}$ is an eigenvector. Thus:

$$
S_{i} \cdot D_{i}^{a}=D_{i}^{a}\left\{[\bar{\rho}]^{-1} \overline{C_{k 1}^{01 p} D_{k}^{a} \cdot \overline{\rho\left(X_{p}^{1 i 1}-X_{i}^{1 p 1}\right)}}\right\}=-\omega^{2}\left[k_{a}\right]^{-2} D_{i}^{a} D_{p}^{a} \overline{\rho\left(X_{p}^{1 i 1}-X_{i}^{1 p .1}\right)}
$$

From which we deduce the orthogonality of the source and the direction of polarization:

$$
S_{i} D_{i}^{a}=0
$$

We have to remark that this property is only valid at the first order. As a matter of fact, supplementary terms appear in the relation between $C^{i}$ and $\rho X^{i}$ when $i>1$. As a result the polarizations of the wave and the diffracted volumic forces are not perpendicular any longer. 